\title{
Hybride textuelle Strukturen und hybride textuelle Einheiten. Ein Beitrag zur Theorie der Wörterbuchform
}

\author{
Herbert Ernst Wiegand, Germanistisches Seminar, Universität Heidelberg, \\ Heidelberg, Bundesrepublik Deutschland \\ (herbert.ernst.wiegand@gs.uni-heidelberg.de)
}

Zusammenfassung: In diesem Beitrag wird die Bildung, Darstellung und Leistung von hybriden textuellen Strukturen, die akzessive Einträge aufweisen, am Beispiel von Wörterbuchartikeln behandelt sowie erklärt, welche Eigenschaften hybride textuelle Einheiten haben. Ein Wörterbuchartikel eines Printwörterbuchs weist immer dann neben einer hierarchischen reinen eine hierarchische hybride Textkonstituentenstruktur auf, wenn in ihm mindestens ein funktionaler Angabezusatz auftritt, z.B. ein oben oder unten erweiternder oder ein binnenerweiternder. Da funktionale Angabezusätze Textsegmente mit Angabefunktion aber ohne Textkonstituentenstatus sind, werden sie durch nichtfunktionale Segmentation ermittelt, so dass neben funktionalen auch nichtfunktionale Textsegmente gegeben sind, die dann bei der Strukturbildung in die Trägermengen eingehen, so dass die Trägermengen aller hybriden hierarchischen Strukturen elementenheterogen, während die Trägermengen aller hierarchischen reinen Strukturen elementenhomogen sind. In den Strukturgraphen für hierarchische hybride Artikelstrukturen sind dann die Knoten für diejenigen Textsegmente, die den Hybridstatus der Strukturen bewirken, entweder durch Pfeilkanten für die textarchitektonischen oberhalb/unterhalb-Relationen mit den Knoten für die Textkonstituenten verbunden, so dass die Strukturgraphen architektonisch angereichert sind, oder durch besonders markierte Kanten, die die Knoten für die nichtfunktionalen Textsegmente und die für die binnenerweiternden funktionalen Angabezusätze mit den Knoten für die Textkonstituenten verbinden. $\mathrm{Zu}$ jedem Typ von hierarchischer reiner Artikelstruktur gehören mehrere Typen von hybriden Artikelstrukturen; entsprechendes gilt für hierarchische reine Angabestrukturen. Nur eine Auswahl aus den Typologien der hybriden Artikel- und Angabestrukturen wird behandelt sowie eine kleine Auswahl von hybriden textuellen Einheiten, die kriteriale Eigenschaften von zwei Textsegmentklassen aufweisen (vgl. die Stichwörter).

Stichwörter: ANGABERELATION, ELEMENTENHETEROGENE TRÄGERMENGE, FUNKTIONALER ANGABEZUSATZ, FUNKTIONAL-POSITIONALE SEGMENTATION, HIERARCHISCHE ARCHITEKTONISCH ANGEREICHERTE ARTIKELMIKROSTRUKTUR, HIERARCHISCHE HYBRIDE ANGABENKONSTITUENTENSTRUKTUR MIT GLOSSATBEDINGTER TEILSTRUKTUR, HIERARCHISCHE HYBRIDE ANGABENSTRUKTUR, HIERARCHISCHE HYBRIDE ARTIKELKONSTITUENTENSTRUKTUR, HIERARCHISCHE HYBRIDE ARTIKELMIKROSTRUKTUR, HIERARCHISCHE HYBRIDE EXHAUSTIVE ANGABENSTRUKTUR, HIERARCHISCHE HYBRIDE GLOSSATBEDINGTE ANGABESTRUKTUR, HIERARCHISCHE HYBRIDE FLACHE 
DOPPELGLOSSATBEDINGTE ANGABESTRUKTUR, HIERARCHISCHE HYBRIDE MINIMIERTE GLOSSATBEDINGTE ANGABESTRUKTUR, HIERARCHISCHE HYBRIDE TEXTKONSTITUENTENSTRUKTUR, HIERARCHISCHE HYBRIDE TIEFE DOPPELGLOSSATBEDINGTE ANGABESTRUKTUR, HIERARCHISCHE REINE TEXTKONSTITUENTENSTRUKTUR, HYBRIDE VERWEISKENNZEICHNUNG, NICHTFUNKTIONALE-POSITIONALE SEGMENTATION, ORDNUNGSRELATION, SEGMENTATIVE ISOLIERUNG, VERTIKALE ANGABEARCHITEKTUR

\begin{abstract}
Hybrid textual structures and hybrid textual units. A contribution to the theory of dictionary structures. In this contribution, the formation, presentation and performance of hybrid textual structures that display accessible entries are discussed by using examples from dictionary articles. The features of hybrid textual units are also explained. A dictionary article in a printed dictionary always displays both a hierarchical pure and a hierarchical hybrid text constituent structure, when it contains at least one functional item addition, e.g. an upward- or downward- or an internally-expanded one. Because functional item additions are text segments with an item function but without text constituent status, they are enabled by means of non-functional segmentation, so that both functional and non-functional text segments prevail. During the formation of structures they then enter the structure-carrying set so that the structurecarrying set of all hybrid hierarchical structures are element-heterogeneous whilst the structurecarrying set of all hierarchical pure structures are element-homogeneous. In the structural diagrams of hierarchical hybrid article structures, the nodes for those text segments that establish the hybrid status of the structures are connected with the nodes for the text constituents either by means of arrows for the text-architectonic upward/downward relations, so that the structural graphs are architectonically enriched, or by means of specially marked edges that connect the nodes for the non-functional text segments and those for the internally-expanded functional item additions with the nodes for the text constituents. To each type of hierarchical pure article structure belong various types of hybrid article structures. The same applies to hierarchical pure item structures. Only a selection from the typology of hybrid article and item structures are discussed as well as a small selection of hybrid textual units that display determining features of two text segment classes (cf. the keywords).
\end{abstract}

Keywords: ELEMENT-HETEROGENEOUS STRUCTURE-CARRYING SET, FUNCTIONAL ITEM ADDITION, FUNCTIONAL-POSITIONAL SEGMENTATION, HIERARCHICAL ARCHITECTONICALLY ENRICHED ARTICLE MICROSTRUCTURE, HIERARCHICAL HYBRID ARTICLE MICROSTRUCTURE, HIERARCHICAL HYBRID DEEP DOUBLE GLOSS-CONDITIONED ITEM STRUCTURE, HIERARCHICAL HYBRID EXHAUSTIVE ITEMS STRUCTURE, HIERARCHICAL HYBRID GLOSS-CONDITIONED ITEM STRUCTURE, HIERARCHICAL HYBRID ITEMS CONSTITUENT STRUCTURE WITH GLOSS-CONDITIONED PARTIAL STRUCTURE, HIERARCHICAL HYBRID ITEMS STRUCTURE, HIERARCHICAL HYBRID MINIMISED GLOSSCONDITIONED ITEM STRUCTURE, HIERARCHICAL HYBRID SHALLOW DOUBLE GLOSSCONDITIONED ITEM STRUCTURE, HIERARCHICAL HYBRIDARTICLE CONSTITUENT STRUCTURE, HIERARCHICAL HYBRIDTEXT CONSTITUENT STRUCTURE, HIERARCHICAL PURE TEXT CONSTITUENT STRUCTURE, HYBRID CROSS-REFERENCE MARKER, ITEM RELATION, NON-FUNCTIONAL-POSITIONAL SEGMENTATION, ORDERING RELATION, SEGMENTATIVE ISOLATION, VERTICAL ITEM ARCHITECTURE 


\section{Vorbemerkung: Was meint das Prädikat hybrid?}

Dt. Hybride ist nach lat. hybrida 'Mischling' gebildet; das zugehörige deutsche Adjektiv hybrid weist ein Bedeutungsspektrum auf, das nach Duden- ${ }^{4} \mathrm{GFWb}$ wie folgt paraphrasiert werden kann: "gemischt, von zweierlei Herkunft, aus Verschiedenem zusammengesetzt; durch Kreuzung, Mischung entstanden". Besonders zutreffend ist hier zunächst die Bedeutungsparaphrasenangabe von zweierlei Herkunft, und zwar aus folgendem Grund: Die Herkunft der Einheiten der Theorie der Wörterbuchform ist immer das Ergebnis einer korrekten Methodenanwendung. Sind die textuellen Einheiten, mit denen die Theorie der Wörterbuchform arbeitet und die im Zuge der Strukturbildung zu einer Trägermenge zusammengefasst werden, das Ergebnis der Anwendung mindestens zweier verschiedener metalexikographischer Methoden, dann gelten die textuellen Strukturen als hybrid. Sind die Elemente der Trägermenge dagegen das Ergebnis der Anwendung nur einer Methode, dann gelten die Strukturen als rein (oder nichthybrid). Nicht nur textuelle Strukturen, sondern auch textuelle Einheiten können hybrid sein. Dies ist stets dann der Fall, wenn eine Einheit eine Eigenschaft aufweist, die zu den kriterialen Eigenschaften der textuellen Einheiten einer anderen Klasse gehört, aber wegen ihrer restlichen Eigenschaften dieser anderer Klassen nicht zugeordnet werden kann (vgl. Wiegand 2010).

\section{Zum Zusammenwirken von Methoden, Theorieausschnitten und Ter- minologie}

Zur Theorie der Wörterbuchform gehört eine Heuristik (vgl. Wiegand 2010), mit der u.a. auch mehrere Segmentationsmethoden für die Segmentation kondensierter lexikographischer Texte bereitgestellt werden (vgl. z.B. Wiegand 1990: 20-26; 2005: 217-226; Wiegand und Fuentes Morán 2009). Für die Anwendung der Segmentationsmethoden sind Anwendungskonventionen und Korrektheitsbedingungen festgelegt. Für die wissenschaftstheoretisch notwendige Verknüpfung von Methoden und Theorieausschnitten gilt neben anderen das folgende Prinzip: Als Elemente für konkrete textuelle Wörterbuchstrukturen kommen ausschließlich solche Textsegmente in Betracht, die durch die korrekte Anwendung einer der Segmentationsmethoden erhältlich sind. Es gelten folgende Unterscheidungen:

(i) Durch eine Anwendung der Methode der exhaustiven funktional-positionalen Segmentation, die eine der Varianten der Methode der funktional-positionalen Segmentation darstellt, sind alle Arten von Textkonstituenten erhältlich, also nur solche funktionalen Textsegmente, die eine diskrete kontinuierliche Textsegmentform, mindestens eine genuine Textsegmentfunktion und genau eine feste textuelle Position innerhalb (und nicht oberhalb oder unterhalb) der sprachlichen Kette des Textes 
aufweisen; dies sind die Folgenden: Angaben, Angabetexte und nichttypographische Strukturanzeiger (vgl. z.B. Wiegand 2009: 254ff; 2009a: 65ff).

(ii) Durch eine Anwendung der Methode der nichtfunktional-positionalen Segmentation sind alle nichtfunktionalen Textsegmente sowie alle binnenerweiternden funktionalen Angabezusätze erhältlich (vgl. z.B. Wiegand 2006: 201ff; 2009: 268ff).

(iii) Durch eine Anwendung der Methode der funktionalen segmentativen Isolierung sind alle oben und unten erweiternden funktionalen Angabezusätze, also die ohne eigene Position in der sprachlichen Kette, erhältlich (vgl. z.B. Wiegand 2006: 193ff; 2009: 270ff).

Es gelten weiterhin die folgenden Festlegungen:

(a) Konkrete hierarchische Textkonstituentenstrukturen, deren Trägermengen in dem Sinne elementenhomogen sind, dass sie als Elemente nur Textsegmente mit Textkonstituentenstatus aufweisen (vgl. (i)) heißen konkrete reine (oder: nichthybride) Textkonstituentenstrukturen: Alle ihre Elemente sind in dem Sinne von gleicher Herkunft, dass sie durch Anwendungen der gleichen metalexikographischen Methode erhältlich sind. Entsprechend heißen abstrakte hierarchische Textkonstituentenstrukturen, deren Trägermengen nur Klassen von Textsegmenten mit Textkonstituentenstatus aufweisen, abstrakte reine (oder: nichthybride) Textkonstituentenstrukturen.

(b) Konkrete hierarchische Textkonstituentenstrukturen, deren Trägermengen in dem Sinne elementenheterogen sind, dass sie als Elemente sowohl Textsegmente mit als auch solche ohne Textkonstituentenstatus aufweisen (vgl. (i) bis (iii)), heißen konkrete hybride Textkonstituentenstrukturen: Ihre Elemente sind in dem Sinne von verschiedener Herkunft (vgl. die in 1. zitierte Paraphrasierung im Duden- ${ }^{4} \mathrm{GFWb}$ ), dass sie durch Anwendungen verschiedener metalexikographischer Methoden erhältlich sind. Entsprechend heißen abstrakte hierarchische Textkonstituentenstrukturen, deren Trägermengen sowohl Klassen von Textsegmenten mit Textkonstituentenstatus als auch Klassen von Textsegmenten ohne Textkonstituentenstatus aufweisen, abstrakte hybride Textkonstituentenstrukturen. - Es kann daher festgestellt werden: Kriterial für die Unterscheidung von konkreten hierarchischen reinen und konkreten hierarchischen hybriden Textkonstituentenstrukturen ist die Zusammensetzung der Trägermengen: Konkrete hierarchische reine Textkonstituentenstrukturen weisen elementenhomogene, konkrete hierarchische hybride Textkonstituentenstrukturen weisen elementenheterogene Trägermengen auf. Für die abstrakten Strukturen gelten diese Zuordnungen mutatis mutandis. Ergänzt sei: Auch hierarchische textuelle Strukturen, die keine Text- 
konstituentenstrukturen sind, wie z.B. bestimmte Angabestrukturen, werden mittels des gleichen Kriteriums in reine und hybride Strukturen unterteilt (vgl. 5).

\section{Reine artikelinterne Textkonstituentenstrukturen}

In kondensierten Wörterbuchartikeln (sensu Wiegand 2003: 203ff) treten reine Textkonstituentenstrukturen auf, die zu fünf Typen gehören: zum Typ der hierarchischen reinen Artikelkonstituentenstruktur, zum Typ der hierarchischen reinen Artikelmikrostruktur, zum Typ der hierarchischen reinen exhaustiven Angabenstruktur, zum Typ der hierarchischen reinen Angabenstruktur und zum Typ der hierarchischen artikelinternen Suchbereichsstruktur (sensu Wiegand 2000: 269ff). Letztere werden in diesem Beitrag nicht berücksichtigt. In diesem Abschnitt wird zunächst ein Beispiel für hierarchische reine Artikelmikrostrukturen in etwas vereinfachter Weise behandelt; auf die Grundlagen der Theorie kann dabei allerdings nicht eingegangen werden (vgl. dazu Wiegand 1989 u. 1989a). Die Darstellung in diesem Abschnitt dient lediglich dem Zweck, den grundlegenden Unterschied zwischen reinen und hybriden textuellen Strukturen genauer zu verstehen; sie dient außerdem dazu, auch anhand eines Beispiels eine größere Anschaulichkeit zu erreichen. Gegeben seien die folgenden Wörterbuchartikel:

wa 1 Smoking, der; -s, -s meist schwarzes Herrenjackett mit seidenen Rockauschlägen für festliche Anlässe: im S. erscheinen

wa2: Streber, der; -s, - jmd., der bes. in der Ausbilding ehrgeizig, aber egoistisch auf sein Fortkommen hinarbeitet: ein gewissenloser, widerlicher S.

wa ${ }_{3}: \quad$ Skale, die; -, -n (bezifferte) Maßeinteilung an Meßgeräten: einen Meßwert an einer S. ablesen

Wa4: Sonate, die; -, -n Komposition mit meist drei od. vier Sätzen für ein od. mehrere Soloinstrumente: sie spielten eine S. für Violine und Klavier von Beethoven

wa5: Spezialist, der; -en, -en jmd., der über besondere Kenntnisse, Fähigkeiten auf einem Fachgebiet verfügt, Fachmann: er ist S. für Halbleitertechnik; er muß einen Spezialisten (Fachartzt) aufsuchen

wa 6 Sweiß, der; -es. /o. Pl./ 1. bes. bei Hitze aus den Poren der Haut austretende wäßrige Absonderung bestimmter Drüsen: der S. steht ihm auf der Stirn, brach ihm aus allen Poren; in S. kommen, geraten; $\diamond$ das hat viel S. (Mühe, Anstrengung) gekostet 2. Jägerspr. Blut des Wildes und des Hundes 
+ im Schweiße seines Angesichts (mit großer Mühe, Anstrengung)

wa7: Bub, der; -en, en süddt. österr. schweiz. Knabe, Junge: ein kleiner, wilder B.; ein B. von fünf Jahren

Abb. 1: Wörterbuchartikel wa ${ }_{1}$ bis wa 7 aus HWDG

Im Folgenden betrachten wir zunächst die konkrete hierarchische reine Artikelmikrostruktur von wa . $_{1}$ Zur Trägermenge jeder Art von konkreter hierarchischer Artikelmikrostruktur gehören keine nichttypographischen Mikrostrukturanzeiger; vielmehr gehören Letztere zur Trägermenge von konkreten hierarchischen Artikelkonstituentenstrukturen, deren prominenteste Substrukturen die konkreten hierarchischen Artikelmikrostrukturen sind sowie zur Trägermenge von konkreten hierarchischen exhaustiven Angabenstrukturen. Demgemäß muss wa ${ }_{1}$, damit seine konkrete hierarchische Artikelmikrostruktur erhältlich ist, in einem ersten methodischen Schritt so segmentiert werden, dass alle Textkonstituenten gegeben sind außer den nichttypographischen Mikrostrukturanzeigen. Da wa ${ }_{1}$ keinen Angabetext aufweist, sind dies nur elementare und nichtelementare Angaben. Daher wird auf wa ${ }_{1}$ die Methode der nichtexhaustiven funktional-positionalen Segmentation angewandt, die eine zweite Variante der Methode der funktional-positionalen Segmentation darstellt und zu deren Korrektheitsbedingungen es gehört, dass als Segmentationsergebnisse nur die Angaben gelten. Es gilt die Segmentationskonvention $\mathrm{K}_{1}$, die besagt, dass Divise (wie z.B. die in den beiden Angaben ",- $\mathrm{s}$ “ in $\mathrm{wa}_{1}$ ) als Teile der Angabeform von Angaben mit Kohäsionsanweisung gelten. Eine andere Segmentationskonvention $\mathrm{K}_{2}$, die hier nicht in Geltung gesetzt wird, könnte lauten, dass die beiden Divise als unmittelbare Teilangaben, nämlich als verdichtete Wortstammangaben gelten sollen, so dass die beiden "-s" in wa keine elementaren, sondern nichtelementare Angaben wären.

Im Folgenden wird das Segmentationsprocedere nicht im Detail beschrieben (vgl. dazu z.B. Wiegand 2000: 235ff u. 2009: 256ff), sondern nur das Segmentationsergebnis so aufgelistet, dass jeder elementaren und jeder nichtelementaren Angabe, die wa ${ }_{1}$ aufweist, zugleich ein Kleinbuchstabe, der in runden Klammern voran steht, als Angabename zugeordnet wird, wobei zu beachten ist, dass Angabenamen Individuennamen sind; weiterhin wird jede Angabe der Klasse von Angaben mit gleicher allgemeiner wörterbuchgegenstandsbezogener genuiner Angabefunktion zugeordnet, zu der sie gehört. Letzteres geschieht mittels Aussagen der Form " $\alpha \in \beta^{\prime \prime}$, mit " $\alpha$ " als Variable für Angabenamen und „ $\beta "$ als Variable für Klassensymbole, die Abkürzungen von Angabeklassennamen sind; dabei ist " $\epsilon$ " das Symbol für die Element-KlassenRelation in der Bedeutung ist ein Element von oder in der Bedeutung von ist enthalten in.

(a) Smoking; der; $-\mathrm{s},-\mathrm{s}\left[\mathrm{i}_{\mathrm{A}} \mathrm{AB}_{\mathrm{j}}\right] ; \mathrm{a} \in \mathrm{FK}$ (= Formkommentar); $\mathrm{AB}=$ Angabeblank. 
(b) Smoking; $\mathrm{b} \in \mathrm{LZGA} \perp$ WAk $\mid$ VQK.L $\mid$ WFA.NSg $\mid$ RA (= Lemmazeichengestaltangabe, ausgeprägt als lemmatische Substantivangabe, unten erweitert durch eine Wortakzentkennzeichnung (WAk), die zugleich $\left({ }^{\prime \prime} \mid\right.$ ') eine Vokalquantitätskennzeichnung zur Länge (VQK.L) ist, weiterhin zugleich Wortformangabe für den Nominativ Singular (WFA.NSg) und zugleich Rechtschreibangabe (RA)).

(c) der; -s, -s; c $\in$ MorA.S (= Morphologieangabe bei Substantiven).

(d) der; $d \in \operatorname{ArtA} \dashv \mathrm{G} \mid \mathrm{WAr}$ (= Artikelangabe, anhand derer das Genus (= G) und zugleich die Wortart (= WAr) erschließbar ist).

(e) $-\mathrm{s},-\mathrm{s} ; \mathrm{e} \in \operatorname{DekKA}$ (= Deklinationsklassenangabe).

(f) $-\mathrm{s} ; \mathrm{f} \in \mathrm{v} \cdot \operatorname{SgbA}$ (= verdichtete Singularbildungsangabe).

(g) $-\mathrm{s} ; \mathrm{g} \in \mathrm{v} \cdot \mathrm{PlbA}$ (= verdichtete Pluralbildungsangabe).

(h) $\quad\left[{ }_{i} A_{\mathrm{j}}\right] ; \mathrm{h} \in \mathrm{A}-\mathrm{rAus}$ (= Angabe zur regelmäßigen Aussprache; diese ist eine Nullangabe, die in der konkreten Struktur durch einen Angabeblank $\left(\mathrm{i}_{\mathrm{i}} \mathrm{B}_{\mathrm{j}}\right)$ repräsentiert wird, der durch die Belegung seiner beiden Nachbarschaftsvariablen,$i^{\prime \prime}$ und,$i^{\prime \prime}$ auch positional identifiziert ist).

(i) meist schwarzes Herrenjackett mit seidenen Rockaufschlägen für festliche Anlässe: im S. erscheinen; i $\in$ SK (semantischer Kommentar).

(j) $\quad\left[{ }_{i} A B_{j}\right] ; j \in$ MonA (= Monosemieangabe; diese ist auch eine Nullangabe, die in der konkreten Struktur durch einen Angabeblank $\left({ }_{i} A B_{j}\right)$ repräsentiert wird, der durch die Belegung seiner beiden Nachbarschaftsvariablen "i" und "i" positional identifiziert ist).

(k) Meist schwarzes Herrenjackett mit seidenen Rockaufschlägen für festliche Anlässe: im S. erscheinen; $\mathrm{k} \in \mathrm{SSK}$ (= semantischer Subkommentar).

(l) $\quad\left[{ }_{i} \mathrm{AB}_{\mathrm{j}}\right]$; meist schwarzes Herrenjackett $[\ldots]$ Anlässe; $1 \in$ PragsemA (= pragmatisch-semantische Angabe).

(m) $\quad\left[\mathrm{i}_{\mathrm{A}} \mathrm{B}\right] ; \mathrm{k} \in \mathrm{A}$-pragNM (= Angabe zur pragmatischen Nullmarkierung; diese ist ebenfalls eine Nullangabe).

(n) Meist schwarzes Herrenjackett [...] Anlässe; $\mathrm{n} \in \mathrm{BPA}$ (= Bedeutungsparaphrasenangabe).

(o) im S. erscheinen; o $\in$ v.BeiA (= verdichtete Kompetenzbeispielsangabe).

Die Angaben b, d, f, g, h, j, m, n und o sind elementare Angaben, also solche, die nicht weiter funktional-positional segmentierbar sind. Nullangaben gelten als elementare Angaben, die auslagerungsresistent sind. Die Angaben a, c, e, i, $\mathrm{k}, 1$ sind nichtelementare Angaben; sie sind mithin restfrei funktional-positional segmentierbar. 
In einem zweiten methodischen Schritt bilden wir nun die elementenhomogene Trägermenge für $\mathrm{MiS}_{\mathrm{h}}^{\mathrm{k}}\left(\mathrm{wa}_{1}\right)$, die konkrete $(\mathrm{k})$ hierarchische $(\mathrm{h})$ reine $(\mathrm{r})$ Artikelmikrostruktur (MiS) von wa ${ }_{1}$; es handelt sich bei der Trägermenge um die um den ganzen Wörterbuchartikel wa ${ }_{1}$ erweiterte Menge aller methodisch ermittelnden Angaben a bis o. Die Trägermenge heiße $\mathrm{M}_{\mathrm{T} M i s}^{\mathrm{k}}\left(\mathrm{wa}_{1}\right)$; sie kann wie folgt mit einer Mächtigkeit von $|16|$ angegeben werden:

$$
\mathrm{M}_{\mathrm{TMiS}}^{\mathrm{k}}\left(\mathrm{wa}_{1}\right)=\left\{w_{1} \mathrm{a}_{1}, \mathrm{a}, \mathrm{b}, \mathrm{c}, \mathrm{d}, \mathrm{e}, \mathrm{f}, \mathrm{g}, \mathrm{h}, \mathrm{i}, \mathrm{j}, \mathrm{k}, \mathrm{l}, \mathrm{m}, \mathrm{n}, \mathrm{o}\right\} .
$$

Dass wa ${ }_{1}$ ein Element von $\mathrm{M}_{\mathrm{TMiS}}^{\mathrm{k}}\left(\mathrm{wa}_{1}\right)$ ist, macht die Trägermenge nicht $\mathrm{zu}$ einer elementenheterogenen Menge, denn auch wa $\mathrm{a}_{1}$ ist eine Textkonstituente, die durch eine Anwendung der Methode der nichtexhaustiven funktionalenpositionalen Segmentation auf die Artikelstrecke $S$ erhältlich ist. Außerdem kann wa ${ }_{1}$ in einem formalen Sinn als die größte Angabe aufgefasst werden.

In einem dritten methodischen Schritt definieren wir auf $\mathrm{M}_{\mathrm{TMiS}}^{\mathrm{k}}\left(\mathrm{wa}_{1}\right)$ eine zweistellige irreflexive (und damit asymmetrische) sowie transitive Relation vom Typ der Präzedenzrelation - sie heiße $\mathrm{R}_{\mathrm{p}}^{\mathrm{k}}\left(\mathrm{wa}_{1}\right)$ - mit dem Relationsterm $s$ geht voraus $y$, mit ", $x$ " und " $y^{\prime \prime}$ als Variable für Angaben. $\mathrm{R}_{\mathrm{p}}^{\mathrm{k}}\left(\mathrm{wa}_{1}\right)$ gehört $\mathrm{zu}$ den irreflexiven partiellen Ordnungsrelationen. Es gilt: $\mathrm{R}_{\mathrm{p}}^{\mathrm{k}}\left(\mathrm{wa}_{1}\right) \subseteq$ $\mathrm{M}_{\mathrm{TMik}}^{\mathrm{k}}\left(\mathrm{wa}_{1}\right)$ x $\mathrm{M}_{\mathrm{TMik}}^{\mathrm{k}}\left(\mathrm{wa}_{1}\right)$. Die strukturprägende Relation $\mathrm{R}_{\mathrm{p}}^{\mathrm{k}}\left(\mathrm{wa}_{1}\right)$ enthält als Elemente alle diejenigen 2-Tupel, wie z.B. $\langle\mathrm{a}, \mathrm{d}\rangle$ und $\langle\mathrm{f}$, g $>$, die - wenn deren Koordinaten in den Relationsterm $x$ geht voraus $y$ eingesetzt werden - wahre Sätze liefern, wie z.B. a geht voraus $d(\approx$ Smoking geht voraus der; mit " $\approx *$, in der Bedeutung von entspricht). $\mathrm{R}_{\mathrm{p}}^{\mathrm{k}}\left(\mathrm{wa}_{1}\right)$ legt damit vollständig fest, welche Angaben von wa ${ }_{1}$ welchen anderen Angaben von wa ${ }_{1}$ vorausgehen. Entsprechendes gilt auch von anderen Relationen (bei späteren Beispielen), die zum Typ der Präzedenzrelation gehören. Präzedenzrelationen heißen auch Vorgänger-Nachfolger-Relationen.

In einem vierten methodischen Schritt definieren wir auf der Trägermenge $\mathrm{M}_{\mathrm{TMiS}}^{\mathrm{k}}\left(\mathrm{wa}_{1}\right)$ eine zweistellige reflexive, antisymmetrische (oder: identitive) und transitive Relation vom Typ der partitiven Relation - sie heiße $\mathrm{R}_{\text {part }}^{\mathrm{k}}\left(\mathrm{wa}_{1}\right)$ - mit dem Relationsterm $x$ ist eine Angabe als Teil von $y$, mit ", $x$ " als Variable für Angaben und " $y "$ als Variable für Angaben und $\mathrm{wa}_{1}$. Es gelten die Aussagen: $\mathrm{R}_{\text {part }}^{\mathrm{k}}\left(\mathrm{wa}_{1}\right) \subseteq \mathrm{M}_{\mathrm{TMik}}^{\mathrm{k}}\left(\mathrm{wa}_{1}\right) \times \mathrm{M}_{\mathrm{TMik}}^{\mathrm{k}}\left(\mathrm{wa}_{1}\right)$ und $\mathrm{R}_{\mathrm{p}}^{\mathrm{k}}\left(\mathrm{wa}_{1}\right) \cap \mathrm{R}_{\text {part }}^{\mathrm{k}}\left(\mathrm{wa}_{1}\right)=\varnothing$. Die Relation $\mathrm{R}_{\text {part }}^{\mathrm{k}}$ (wa $\mathrm{w}_{1}$ ) gehört $\mathrm{zu}$ den reflexiven partiellen Ordnungsrelationen. Sie enthält als Elemente diejenigen 2-Tupel (oder: geordneten Paare), wie z.B. $<\mathrm{d}, \mathrm{c}>$ und $<\mathrm{a}, \mathrm{wa}_{1}>$, die - wenn deren Koordinaten in den Relationsterm $x$ ist eine Angabe als Teil von y eingesetzt werden - wahre Sätze, wie z.B. $d$ ist eine Angabe als Teil von $c\left(\approx\right.$ "der" ist eine Angabe als Teil von "der" -es, - $\left.-\mathrm{e}^{\prime \prime}\right)$, liefern. Die Relation $\mathrm{R}_{\text {part }}^{\mathrm{k}}\left(\mathrm{wa}_{1}\right)$ legt damit vollständig fest, (i) welche Angaben von wa $a_{1}$ Teilangaben von welchen anderen Angaben von wa ${ }_{1}$ sind, sowie (ii) welche Angaben Teile von wa ${ }_{1}$ sind. Die Vereinigung der beiden strukturprägenden Relationen, $R_{\mathrm{p}}^{\mathrm{k}}\left(w \mathrm{w}_{1}\right) \cup \mathrm{R}_{\mathrm{part}}^{\mathrm{k}}\left(\mathrm{wa}_{1}\right)$, ordnet die Trägermenge vollständig. 
Nach der Ausführung des vierten methodischen Schrittes ist $\mathrm{rMiS}_{\mathrm{h}}^{\mathrm{k}}\left(\mathrm{wa}_{1}\right)$, die reine konkrete hierarchische Artikelmikrostruktur von wa ${ }_{1}$, als eine Ordnungsstruktur gegeben, die festlegt, welchen strukturellen Ort jede Angabe in $\operatorname{rMiS}_{\mathrm{h}}^{\mathrm{k}}\left(\mathrm{wa}_{1}\right)$ aufweist. Damit kann festgestellt werden, dass hierarchische reine Artikelmikrostrukturen von vollständig kondensierten Wörterbuchartikeln (sensu Wiegand 2003: 207f), die keine Angabetexte aufweisen, als artikelinterne Angabedistributionsstrukturen gelten können.

Der fünfte methodische Schritt besteht darin, dass die Definition für die konkrete hierarchische reine Artikelmikrostruktur von $\mathrm{wa}_{1}$ formuliert wird. Dabei muss auf die so genannten Hilfsbasismengen (in der Terminologie von Bourbaki 1957) zurückgegriffen werden. Hilfsbasismengen sind endliche Mengen von Objekten aus dem Bereich, auf den die mathematische Strukturtheorie der Mathematikergruppe Bourbaki angewandt wird. Der Bereich ist hier das HWDG. Für die Analyse von hierarchischen reinen Artikelmikrostrukturen in konkreter und abstrakter Ausprägung benötigt man folgende Hilfsbasismengen:

- $\quad W_{1}$ ist die Menge aller kondensierten Wörterbuchartikel des HWDG; $\left(\right.$ wa $\left._{1} \in \mathrm{WA}_{1}\right)$

- $\quad \mathrm{A}_{1}$ ist die Menge aller Angaben in kondensierten Wörterbuchartikeln des HWDG

- $\quad \mathrm{AK}_{1}$ ist die Menge aller Mengen von Angaben mit gleicher allgemeiner genuiner Funktion, die in kondensierten Wörterbuchartikeln des HWDG gegeben sind; kurz: $\mathrm{AK}_{1}$ ist die Menge aller Angabeklassen. Die Elemente von $\mathrm{AK}_{1}$ sind Äquivalenzklassen über $\mathrm{A}_{1}$, die dadurch gebildet werden, dass auf $\mathrm{A}_{1}$ eine Relation vom Typ der Äquivalenzrelation definiert wird, die transitiv, symmetrisch und reflexiv ist und zu der der Relationsterm $x$ ist mit $y$ gleich hinsichtlich der allgemeinen wörterbuchgegenstandsbezogenen genuinen Funktion gehört, mit " $x$ " und " $y$ " als Variablen für Angaben aus A. Es gilt: $\mathrm{AK}_{1} \subseteq \mathrm{A}_{1}$.

Die Definition lautet dann wie folgt:

Def. 1: $\mathrm{rMiS}_{\mathrm{h}}^{\mathrm{k}}\left(\mathrm{wa}_{1}\right)$, die konkrete (k) hierarchische (h) reine (r) Artikelmikrostruktur (MiS) von wa ist eine Ordnungsstruktur, bestehend aus der Trägermenge $\mathrm{M}_{\mathrm{TMiS}}^{\mathrm{k}}\left(\mathrm{wa}_{1}\right)$, auf der die partitive Relation $\mathrm{R}_{\text {part }}^{\mathrm{k}}\left(\mathrm{wa}_{1}\right)$ sowie die Präzedenzrelation $\mathrm{R}_{\mathrm{p}}^{\mathrm{k}}\left(w \mathrm{w}_{1}\right)$ definiert sind, und für die folgende Beziehungen $\mathrm{zu}$ den Hilfsbasismengen $\mathrm{WA}_{1}, \mathrm{~A}_{1}$ und $\mathrm{AK}_{1}$ gelten:

(i) Ein Element stammt aus $\mathrm{WA}_{1}$.

(ii) Die anderen Elemente stammen aus Elementen von $\mathrm{AK}_{1}$ (also aus den Äquivalenzklassen über $\mathrm{A}_{1}$ ).

Eine formale Form der Def. 1 kann wie folgt angegeben werden: 
Def. $1^{\prime}$ : $\operatorname{rMiS}_{\mathrm{h}}^{\mathrm{k}}\left(\mathrm{wa}_{1}\right)=$ def. $<\mathrm{M}_{\mathrm{TMiS}}^{\mathrm{k}}\left(\mathrm{wa}_{1}\right), \mathrm{A}_{1}, \mathrm{AK}_{1}, \mathrm{WA}_{1} ; \mathrm{R}_{\text {part }}^{\mathrm{k}}\left(\mathrm{wa}_{1}\right)$, $\mathrm{R}_{\mathrm{p}}^{\mathrm{k}}\left(\mathrm{wa} \mathrm{a}_{1}\right)>$.

Bei einer strengen Anwendung der mathematischen Strukturtheorie von Bourbaki gehört zu einer Definition von Ordnungsstrukturen eine Menge von Ordnungsaxiomen; diese sind Bedingungen, die alle konkreten hierarchischen reinen Artikelmikrostrukturen erfüllen müssen, wenn für sie die Def. 1 gelten soll. Informell formuliert lauten solche Bedingungen wie folgt:

(a) Der Formkommentar von wa $a_{1}$ ist eine unmittelbare Textkonstituente von wa 1 und hat keinen Vorgänger bezüglich $\mathrm{R}_{\mathrm{p}}^{\mathrm{k}}\left(\mathrm{wa}_{1}\right)$.

(b) Der semantische Kommentar von wa ${ }_{1}$ ist eine unmittelbare Textkonstituente von wa $\mathrm{a}_{1}$ und hat genau einen Vorgänger bezüglich $\mathrm{R}_{\mathrm{p}}^{\mathrm{k}}\left(\mathrm{wa}_{1}\right)$, nämlich den Formkommentar.

(c) Das aus WA stammende Element wa $a_{1}$ dominiert alle anderen Elemente der Trägermenge.

Die relativ aufwendige formale Formulierung aller Ordnungsaxiome kann man sich sparen, wenn man den Strukturgraphen für $\mathrm{rMiS}_{\mathrm{h}}^{\mathrm{k}}\left(\mathrm{wa}_{1}\right)$ (am besten zusammen mit dem für die zugehörige abstrakte (a) Artikelmikrostruktur $\mathrm{rMiS}_{\mathrm{h}}^{\mathrm{a}}\left(\mathrm{wa}_{1}\right)$ ) angibt. Jeder, der weiß, wie man einen solchen geordneten Strukturgraph, der aus zwei aufeinander abgebildeten geordneten Baumgraphen besteht, richtig liest, kann leicht alle Ordnungsaxiome an diesem ablesen.

Abstrakte Artikelmikrostrukturen sind dadurch erhältlich, dass die beiden strukturprägenden Ordnungsrelationen auf einer Trägermenge definiert werden, die nicht wa ${ }_{1}$ und die konkreten Angaben a bis o enthält, sondern die Klasse $W_{1}$ der Wörterbuchartikel des HWDG sowie die Angabeklassen, zu denen die Angaben a bis o gehören. Die Trägermenge - sie heiße $\mathrm{M}_{\mathrm{TMiS}}^{\mathrm{a}}\left(\mathrm{wa}_{1}\right)$ - kann unter Verwendung der in (a)-(o) eingeführten Klassensymbole entsprechend wie folgt mit einer Mächtigkeit von |16| angegeben werden:

$$
\begin{aligned}
& \mathrm{M}_{\mathrm{TMis}}^{\mathrm{a}}\left(\mathrm{wa}_{1}\right)=\{\mathrm{WA}, \mathrm{FK}, \mathrm{LZGA}\rfloor \text { WAk } \mid \text { VQK.L } \mid \text { WFA.NSg } \mid \text { RA, MorA.S, } \\
& \text { ArtA } \dashv \mathrm{G} \mid \text { WAr, DekKA, v.SgbA, v.PLbA, A-rAus, SK, MonA, SSK, } \\
& \text { PragsemA, A-pragNM, v.BPA, v.KBeiA }\} .
\end{aligned}
$$

Der einfach kommentierte Strukturgraph zur konkreten (und zur isomorphen abstrakten) hierarchischen reinen Artikelmikrostruktur von wa ${ }_{1}$ findet sich in Abb. 2.

Es lassen sich zahlreiche Typen von reinen Mikrostrukturen unterscheiden. Eine (nicht ganz vollständige) Übersicht findet man in Wiegand (2002: 573-580). 


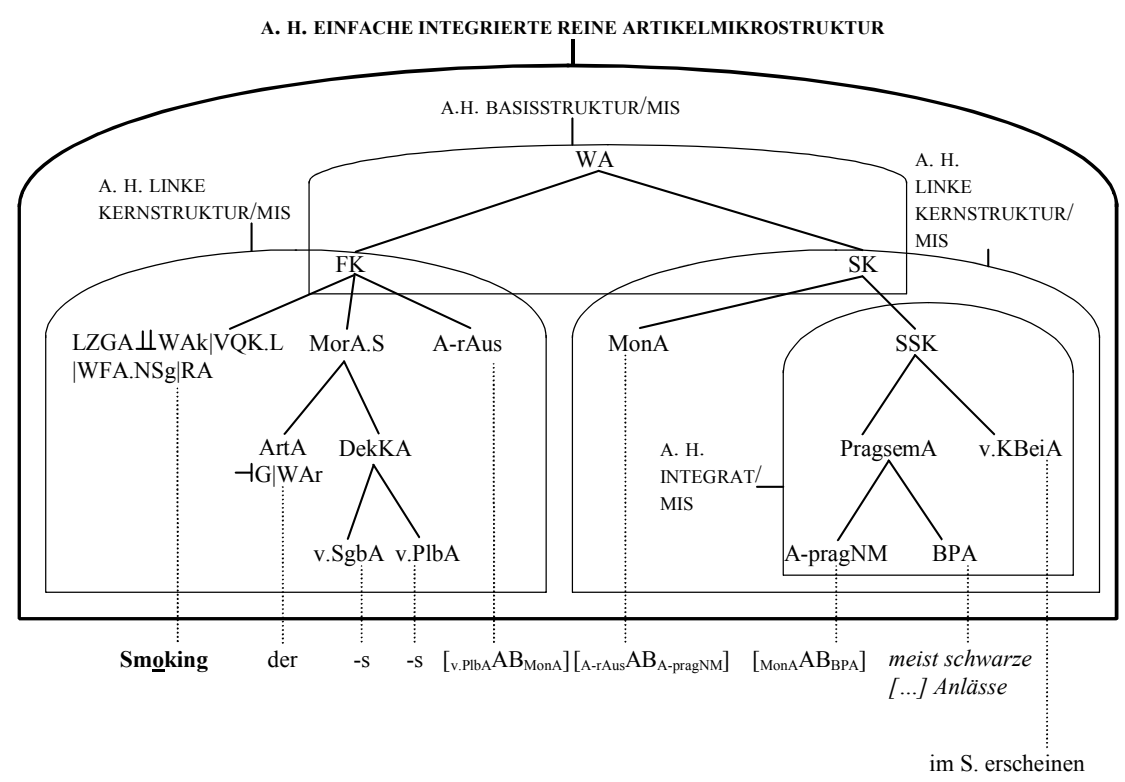

Abb. 2: Einfach kommentierter Strukturgraph zur abstrakten (und isomorphen konkreten) hierarchischen einfachen integrierten reinen Artikelmikrostruktur, die wa ${ }_{1}$ in Abb. 1 aufweist.

Darstellungskonventionen: „, $\mathrm{x}-\mathrm{y}^{\prime \prime}$ bedeutet (von unten nach oben gelesen) soviel wie $x$ ist eine Angabe als Teil von $y_{;}, ", \mathrm{u}^{---} \mathrm{V}^{\prime \prime}$ bedeutet (von unten nach oben gelesen) soviel wie $u$ ist ein Element von $V(=\mathrm{u} \in \mathrm{V}$, wobei "V" ein Klassensymbol ist. "|" bedeutet soviel wie zugleich; LINKE KERNSTRUKTUR/ MIS ist zu lesen wie linke Kernstruktur als Teilstruktur der Artikelmikrostruktur; Abkürzungen (alle Abkürzungen außer WA, mit denen Knoten etikettiert sind, sind Klassensymbole für Klassen von Angaben mit gleicher allgemeiner wörterbuchgegenstandsbezogener genuiner Angabefunktion): A. = ABSTRAKTE; H. = HIERARCHISCHE; WA = Wörterbuchartikel; FK = Formkommentar; SK = semantischer Kommentar; LZGA = Lemmazeichengestaltangabe; WFA.NSg = Wortformenangabe Nominativ Singular; RA = Rechtschreibangabe; MorA.S = Morphologieangabe bei Substantiven; A-rAus = Angabe zur regelmäßigen Aussprache; ArtA = Artikelangabe; DekKA = Deklinationsangabe; v.SgbA = verdichtete Singularbildungsangabe; $\quad$ v.PlbA = verdichtete Pluralbildungsangabe; PragsemA = pragmatisch-semantische Angabe; ApragNM = Angabe zur pragmatischen Nullmarkierung; BPA = Bedeutungsparaphrasenangabe; v.KBeiA = verdichtete Kompetenzbeispielangabe; ${ }_{\mathrm{i}} \mathrm{AB}_{\mathrm{j}}=$ Angabeblank (mit Nachbarschaftsvariablen ${ }_{,}, i^{\prime \prime},{ }^{\prime}, j^{\prime \prime}$, deren Belegung die Identifizierung und Positionierung des $\mathrm{AB}$ sicherstellen)

\section{Hybride artikelinterne Textkonstituentenstrukturen I: Artikelkonsti- tuenten- und Artikelmikrostrukturen}

Hybride Artikelkonstituenten- und Artikelmikrostrukturen lassen sich einem kondensierten Wörterbuchartikel (i.S.v. Wiegand 2003: 200f) dann zuweisen, 
wenn er mindestens einen funktionalen Angabezusatz aufweist. Für Wörterbuchartikel, in denen funktionale Angabezusätze gegeben sind, lässt sich sowohl eine reine als auch mindestens eine hybride Artikelkonstituenten- und Artikelmikrostruktur angeben. Funktionale Angabezusätze sind funktionale Textsegmente, mit denen - wie mit Angaben - etwas angegeben wird, so dass sie eine Angabefunktion haben, die aber - im Unterschied zu Angaben keinen Textkonstituentenstatus aufweisen (vgl. zu funktionalen Angabezusätzen z.B. Wiegand 2005: 326-330; 2007: 192ff); sie sind nicht — wie Angaben als Ergebnis einer funktional-positionalen Segmentation erhältlich, weil sie entweder keine eigene Position in der sprachlichen Kette aufweisen (wie z.B. der Unterstrich unter dem Angabeformsegment ", $\mathbf{0}^{\prime \prime}$ in der Lemmazeichengestaltangabe von $\mathrm{wa}_{1}$ ), oder weil sie, wenn sie eine eigene Position in der sprachlichen Kette aufweisen, nicht funktional-positional isolierbar sind, wie z.B. das semantisch Binnenglossat "Facharzt" in wa5 in Abb.1; denn eine Segmentation führt zu den beiden nichtfunktionalen Textsegmenten „er muß einen Spezialisten" und "aufsuchen", so dass die Segmentation nichtfunktionalpositional ist. Der Unterstrich in wa ${ }_{1}$ gehört zu den unten erweiternden funktionalen Angabezusätzen, und zwar handelt es sich um einen unten erweiternden bifunktionalen Angabezusatz, der an das Angabeformsegment "0 adressiert ist; mit ihm wird eine Wortakzentkennzeichnung realisiert, die zugleich eine Vokalquantitätskennzeichnung zur Länge ist (WAk | VQK.L). Das Gleiche gilt für die Unterstriche in wa $\mathrm{w}_{2}$ bis wa. Weist man wa $\mathrm{w}_{5}$ eine konkrete und eine abstrakte hierarchische reine Artikelmikrostruktur $\mathrm{zu}$, dann sind diese auf die gleiche Weise erhältlich wie die Artikelmikrostrukturen von wa und können wie folgt in Abb. 3 dargestellt werden.

Wenn wir den Strukturgraphen in Abb. 3 aufmerksam betrachten, erkennen wir, dass in der abstrakten hierarchischen Artikelmikrostruktur zu der Klasse, zu der die Wortakzentkennzeichnung, die zugleich eine Vokalquantitätskennzeichnung zur Kürze gehört, kein eigener Knoten gegeben ist. Sie weist damit keinen eigenen strukturellen Ort im geordneten Baumgraphen auf, der mittels mindestens einer Kante mit einem anderen strukturellen Ort des gleichen Baumgraphen verbunden ist. Dass es diesen unten erweiternden

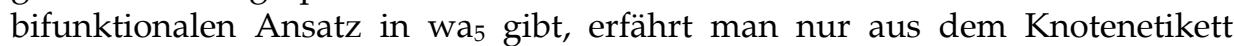
„LZGA $\Perp W A k \mid$ VQK.K $\mid$ WFA.NSg | RA“. Auch in der zugehörigen isomorphen konkreten Artikelmikrostruktur kann daher der Unterpunkt in der unten erweiterten Lemmazeichengestaltangabe keinen eigenen strukturellen Ort aufweisen. Vielmehr erscheint er in "Spezialịst" wie im konkreten Text des Wörterbuchartikels! Will man bei der Darstellung von Artikelmikrostrukturen nicht nur die artikelinterne Angabedistribution und gegebenenfalls die Distribution der Angabetexte explizit berücksichtigen, sondern (falls vorhanden) auch die der funktionalen Angabezusätze, muss man von reinen zu hybriden Mikrostrukturen übergehen. Am Beispiel von wa 5 sei dies nun zunächst stellvertretend für alle unten erweiternden funktionalen Angabezusätze erläutert, ohne dass alle formalen Details berücksichtigt werden. 


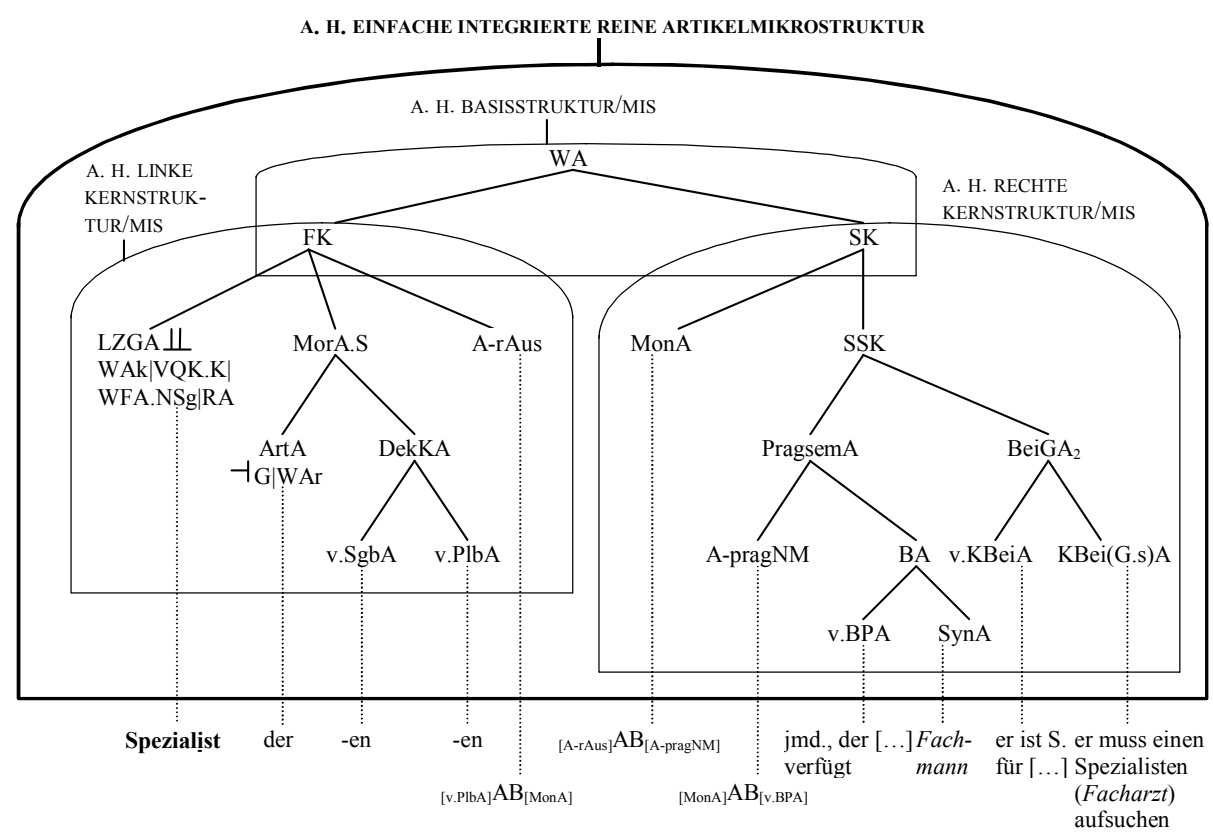

Abb. 3: Einfach kommentierter Strukturgraph zur abstrakten (und isomorphen konkreten) hierarchischen einfachen integrierten reinen Artikelmikrostruktur, die was in Abb. 1 aufweist

Abkürzungen: WAk $\mid \mathrm{VQK} . \mathrm{K}=$ Wortakzentkennzeichnung, zugleich Vokalquantitätskennzeichnung zur Kürze; BeiGA ${ }_{2}=$ Beispielgruppenangabe, die aus zwei Beispielangaben besteht; KBei(G.s)A = um ein semantisches Binnenglossat (G.s) binnenerweiterte Kompetenzbeispielangabe; BA = Bedeutungsangabe; SynA = Synonymangabe

Im Folgenden betrachten wir zunächst die unten erweiterte Lemmazeichengestaltangabe "Spezialịst" aus wa. Sie gehört zu den einfach erweiterten elementaren Angaben und bei diesen zu den unten erweiterten (vgl. Wiegand 2005: 289). Während nichterweiterte elementare Angaben, wie z.B. die Artikelangabe "der" in was, keine interne Struktur aufweisen, ist dies bei erweiterten elementaren Angaben anders: Ihnen kann eine interne Struktur zugewiesen werden. Deren Elemente sind bei oben oder unten erweiterten elementaren Angaben durch eine Anwendung der Methode der funktionalen segmentativen Isolierung erhältlich. Bei der Anwendung dieser Methode werden horizontale Segmentationsschnitte so angelegt, dass die oben oder unten erweiternden funktionalen Angabezusätze von der elementaren Angabe abgetrennt werden. Bei der unten erweiterten Lemmazeichengestaltangabe "Spezialịst" bedeutet dies, dass das Segmentationsergebnis aus dem Segment "Spezialist" und dem Unterpunkt besteht; es ist zu beachten, dass beide Segmente Teile von "Spezialịst" sind. Um die genaue Position des Unterpunktes festlegen zu können, wird 
„Spezialịst" nichtfunktional-positional segmentiert, und zwar so, dass sich folgende Angabeformsegmente ergeben: Spezial $|\mathbf{i}|$ st (mit " $\mid$ " als Segmentationsfugenmarkierung) "Spezial“ ist das vordere, ",i" ist das mittlere und "st" ist das hintere Angabeformsegment. Es gilt die Aussage: Spezial $<\mathbf{i}<\mathbf{s t}$ (mit "< für geht voraus). Mit Angabeformsegmenten werden keine bedeutungstragenden Einheiten genannt; dadurch unterscheiden sie sich von Angabesegmenten, mit denen solche Einheiten genannt werden.

Um die interne Angabestruktur von "Spezialịst" angeben zu können, gehen wir im Folgenden schrittweise vor; wir bilden zunächst folgende Trägermenge $\mathrm{M}_{\mathrm{Ar}}^{\mathrm{k}}$ (Spezialịst) $=\{\mathbf{i}, \bullet\}$; diese Trägermenge enthält also zwei konkrete (k) Elemente: das mittlere Angabeformsegment , $\mathbf{i}^{\prime \prime}$ und den Unterpunkt. Auf $\mathrm{M}_{\mathrm{Ar}}^{\mathrm{k}}$ (Spezialịst) definieren wir nun eine zweistellige irreflexive und damit asymmetrische sowie transitive Relation $\mathrm{R}_{\mathrm{Ar}}^{\mathrm{k}}$ (Spezialịst) vom Typ der (textarchitektonischen) unterhalb-Relation, zu welcher der Relationsterm $x$ ist unterhalb von $y$ gehört, mit " $x$ " als Variable für funktionale Angabezusätze und " $y^{\prime \prime}$ als Variable für deren Bezugsadresse. Die Relation $\mathrm{R}_{\mathrm{Ar}}^{\mathrm{k}}$ (Spezialịst) prägt auf der Trägermenge eine Struktur, die zu den vertikalen Angabearchitekturen zählt, da Strukturen, deren strukturprägende Relationen oberhalb- oder unterhalbRelationen und/oder links-von- und rechts-von-Relationen sind, Architekturen heißen (vgl. z.B. Wiegand 2001: 191ff). Vertikale Angabearchitekturen lassen sich unter Verwendung der gleichen formalen Darstellungsmittel darstellen wie vertikale Textarchitekturen von Wörterbuchartikeln (vgl. zu diesen z.B. Bergenholtz, Tarp und Wiegand 1999: 1791ff $u$. Wiegand 2001: 191ff). Das allgemeine Angabearchitekturbild für die unten erweiterte Lemmazeichengestaltangabe aus wa ${ }_{5}$ findet sich in Abb.4.

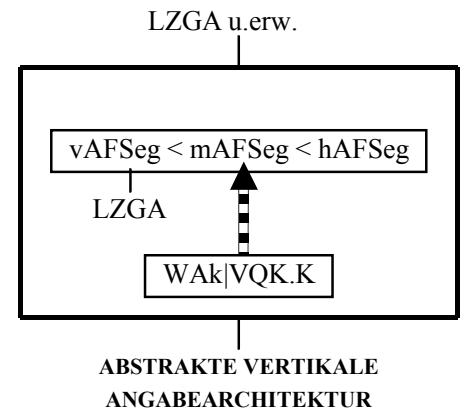

Abb. 4: Allgemeines Angabearchitekturbild zur abstrakten vertikalen Angabearchitektur der unten erweiterten Lemmazeichengestaltangabe aus $\mathrm{wa}_{5}$ Abkürzungen: $\mathrm{vAFSeg}=$ vorderes Angabeformsegment; $\mathrm{mAFSeg}=$ mittleres Angabeformsegment; hAFSeg = hinteres Angabeformsegment; u. erw. = unten erweitert; , $\mathrm{x} \cdots \mathrm{y}^{\prime \prime}$ bedeutet soviel wie $x$ ist unterhalb von $y$, wenn der Pfeil nach oben zeigt 
Im Folgenden erweitern wir die Trägermenge $\mathrm{M}_{\mathrm{Ar}}^{\mathrm{k}}$ (Spezialịst) um „Spezial“, das vordere Angabeformsegment, und weiterhin um "st ${ }^{\prime \prime}$, das hintere Angabeformsegment, sowie um die nichterweiterte Lemmazeichengestaltangabe "Spezialist" und schließlich noch um die unten erweiterte Lemmazeichengestaltangabe "Spezialịst", so dass wir als erweiterte elementenheterogene Trägermenge - sie heiße $\mathrm{M}_{\text {hyAnS }}^{\mathrm{k}}$ (Spezialịst) - eine Menge mit der Mächtigkeit $|6|$ erhalten, die wie folgt angegeben werden kann:

$$
\mathrm{M}_{\text {hyAnS }}^{\mathrm{k}}(\text { Spezialịst })=\{\mathrm{i}, \bullet, \text { st, Spezial, Spezialịst, Spezialist }\} .
$$

Auf dieser elementenheterogenen Trägermenge, die drei Angabeformsegmente, einen funktionalen Angabezusatz und eine nichterweiterte sowie eine unten erweiterte Angabe als Elemente enthält, definieren wir die drei strukturprägenden Relationen, deren zugehörige Relationstypen wir bereits kennen, und zwar zunächst eine Relation vom Typ der Präzedenzrelation - sie heiße $\mathrm{R}_{\mathrm{p}}^{\mathrm{k}}$ (Spezialịst) - mit dem Relationsterm $x$ geht voraus $y$, mit ", $x^{\prime \prime}$ und ", $y^{\prime \prime}$ als Variablen für Textsegmente. Es gilt $\mathrm{R}_{\mathrm{p}}^{\mathrm{k}}$ (Spezialịst) $\subseteq \mathrm{M}_{\text {hyAns }}^{\mathrm{k}}$ (Spezialịst) $\mathrm{x}$ $\mathrm{M}_{\mathrm{hyAnS}}^{\mathrm{k}}$ (Spezialịst). - Weiterhin wird auf $\mathrm{M}_{\mathrm{hyAnS}}^{\mathrm{k}}$ (Spezialịst) eine Relation vom Typ der partitiven Relation - sie heiße $\mathrm{R}_{\text {part }}^{\mathrm{k}}$ (Spezialist) - definiert, mit dem Relationsterm $u$ ist ein Teil von $v$ mit " $u$ " und " $v^{\prime \prime}$ als Variable für Textsegmente. Es gelten:

$$
\begin{aligned}
& \mathrm{R}_{\text {part }}^{\mathrm{k}}(\text { Spezialịst }) \subseteq \mathrm{M}_{\text {hyAnS }}^{\mathrm{k}}(\text { Spezialịst }) \quad \mathrm{x} \quad \mathrm{M}_{\text {hyAnS }}^{\mathrm{k}} \text { (Spezialịst) sowie } \\
& \mathrm{R}_{\text {part }}^{\mathrm{k}}(\text { Spezialịst }) \cup \mathrm{R}_{\mathrm{p}}^{\mathrm{k}}(\text { Spezialịst })=\varnothing \text {. }
\end{aligned}
$$

- Schließlich definieren wir auf $\mathrm{M}_{\mathrm{hyAnS}}^{\mathrm{k}}$ (Spezialịst) eine Relation vom Typ der textarchitektonischen unterhalb-Relation - sie heiße $\mathrm{R}_{\mathrm{unt}}^{\mathrm{k}}$ (Spezialịst) - mit dem Relationsterm $r$ ist unterhalb von $s$, mit " $r^{\prime \prime}$ als Variable für funktionale Angabezusätze und "s" als Variable für deren Bezugsadressen. Es gelten:

$$
\begin{aligned}
& \mathrm{R}_{\text {unt }}^{\mathrm{k}}(\text { Spezialịst })=\mathrm{M}_{\text {hyAnS }}^{\mathrm{k}}(\text { Spezialịst }) \times \mathrm{M}_{\text {hyAnS }}^{\mathrm{k}}(\text { Spezialịst }) ; \mathrm{R}_{\text {unt }}^{\mathrm{k}}(\text { Spezi- } \\
& \text { alịst) } \cap \mathrm{R}_{\mathrm{p}}^{\mathrm{k}}(\text { Spezialịst })=\varnothing ; \quad \mathrm{R}_{\text {unt }}^{\mathrm{k}} \text { (Spezialịst) } \cap \mathrm{R}_{\text {part }}^{\mathrm{k}} \text { (Spezia- } \\
& \text { lịst) }=\varnothing \text {. }
\end{aligned}
$$

Als Ergebnis aller genannten Operationen erhalten wir die konkrete hierarchische architektonisch angereicherte Angabestruktur der unten erweiterten Lemmazeichengestaltangabe aus wa5; diese ist in Abb. 5 (1) dargestellt; in Abb. 5 (2) findet sich die zugehörige isomorphe abstrakte hierarchische architektonisch angereicherte Angabestruktur, die dadurch erhältlich ist, dass man auf der elementenheterogenen Trägermenge

$$
\begin{aligned}
& \mathrm{M}_{\text {hyAnS }}^{\mathrm{a}}(\text { Spezialist })=\{\text { mAFSeg, WAk } \mid \text { VQK.K, vAFSeg, hAFSeg, LZGA } \\
& \qquad \text { WAk } \mid \text { VQK.K }\} .
\end{aligned}
$$

drei Relationen definiert, die zu demselben Relationstyp gehören wie die drei auf $\mathrm{M}_{\text {hyAns }}^{\mathrm{k}}$ (Spezialịst) definierten Relationen. 
(1)

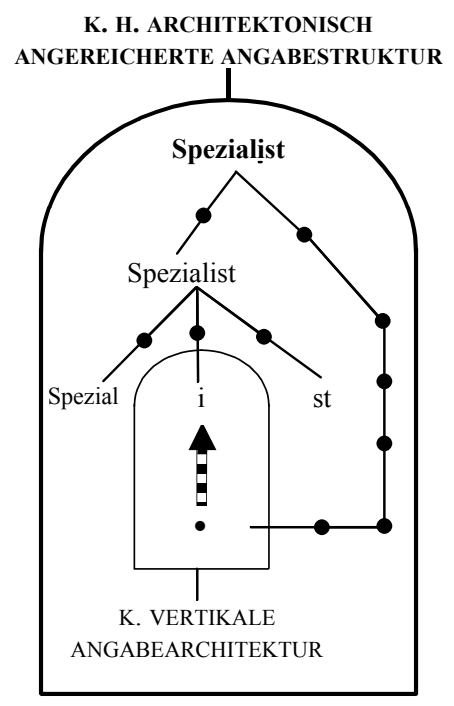

(2)

A. H. ARCHITEKTONISCH ANGEREICHERTE ANGABESTRUKTUR

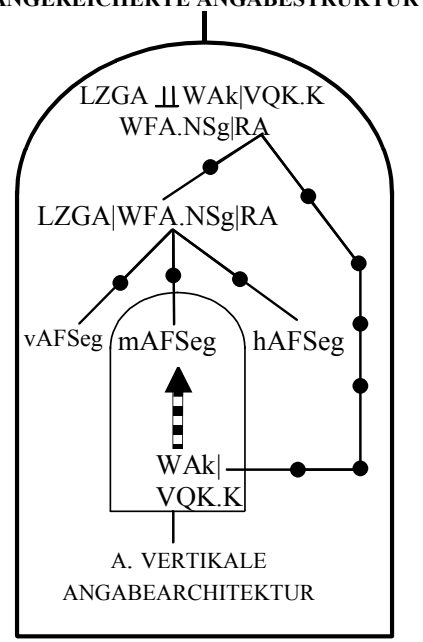

Abb. 5: Einfach kommentierte und mit architektonischen Komponenten angereicherte Baumgraphen zur konkreten (1) und zur isomorphen abstrakten hierarchischen Angabestruktur (2) der unten erweiterten Lemmazeichengestaltangabe aus wa 5

Abkürzungen: $\mathrm{K}=$ KONKRETE; Darstellungskonventionen: „x $\mathrm{x} \rightarrow \mathrm{y}$ “ bedeutet soviel wie $x$ ist unterhalb von $y_{;}$, , $\mathrm{u} \rightarrow-\mathrm{v}^{\prime \prime}$ bedeutet soviel wie $u$ ist ein Teil von $v$, mit ",$u^{\prime \prime}$ als Variable für Textsegmente, die keine Textkonstituenten sind

Die beiden in Abb. 5 präsentierten Baumgraphen lassen sich zu einem Strukturgraphen zusammenfassen; dieser findet sich in Abb. 6.

Weiter oben wurde festgelegt, dass zu jedem Wörterbuchartikel, in dem mindestens ein funktionaler Angabezusatz gegeben ist, sowohl eine reine als auch mindestens eine hybride Artikelmikrostruktur angegeben werden kann. Der Übergang von einer reinen zu einer hybriden Struktur kann auch ausschließlich auf der Ebene der Strukturdarstellung erfolgen, da es sich um strikt formale Strukturdarstellungen handelt. Demgemäß wird nun der Strukturgraph in Abb. 6 in den Strukturgraph in Abb. 3 integriert, so dass sich der Strukturgraph von wa5 ergibt; dieser findet sich in Abb. 7, wobei auf die vollständige Wiedergabe der Kommentarstruktur des semantischen Kommentars verzichtet wurde, weil der funktionale Angabezusatz im semantischen Kommentar von wa 5 noch nicht analysiert ist. 


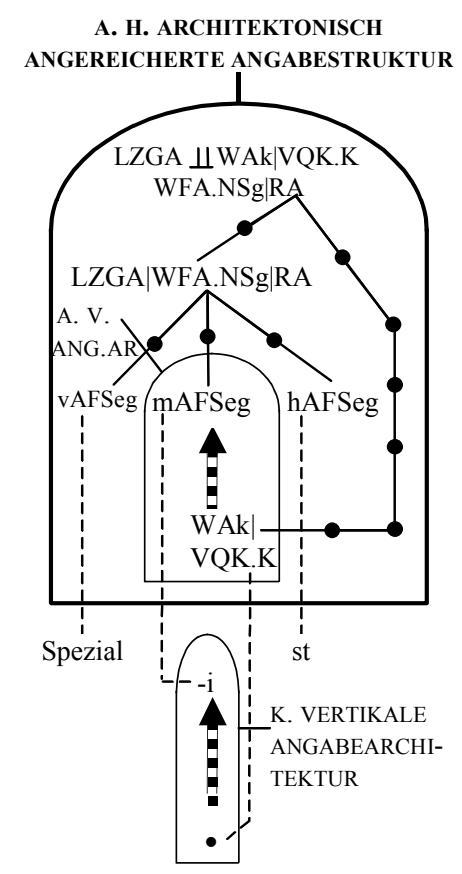

Abb. 6: Einfach kommentierter und mit architektonischen Komponenten angereicherter Strukturgraph zur abstrakten (und isomorphen konkreten) hierarchischen Angabestruktur der unten erweiterten Lemmazeichengestaltnangabe aus $\mathrm{wa}_{3}$

Abkürzungen: A. V. ANG.AR = ABSTRAKTE VERTIKELE ANGABEARCHITEKTUR; Darstellungskonventionen wie in Abb. 5; Darstellungskonventionen: „X----Y" bedeutet (von unten nach oben gelesen) soviel wie $x$ ist ein Element von $Y(=\mathrm{x} \in \mathrm{Y})$

Bisher wurden hybride Angabestrukturen behandelt, die zu dem Typ der hierarchischen architektonisch angereicherten Angabestrukturen gehören, der ein Untertyp des Typs der hierarchischen hybriden konstituentenlosen Angabestruktur ist; der zuletzt genannte Strukturtyp weist einen weiteren Untertyp auf, nämlich den Typ der hierarchischen binnenerweiterungsbedingten Angabestruktur (vgl. Wiegand 2007: 201ff u. Abb. 20). Handelt es sich, wie z.B. in wa5, bei den binnenerweiternden funktionalen Angabezusätzen um Binnenglossate, wird von hybriden binnenglossatbedingten Angabestrukturen gesprochen und verkürzt von hybriden glossatbedingten Angabestrukturen. Der verkürzte Terminus ist ausreichend deutlich, da postglossierte Angaben stets zu den nichtelementaren Angaben gehören, so dass „postglossatbedingte Angabestrukturen" nicht auftreten können. Im Folgenden betrachten wir glossatbedingte Angabestrukturen. 


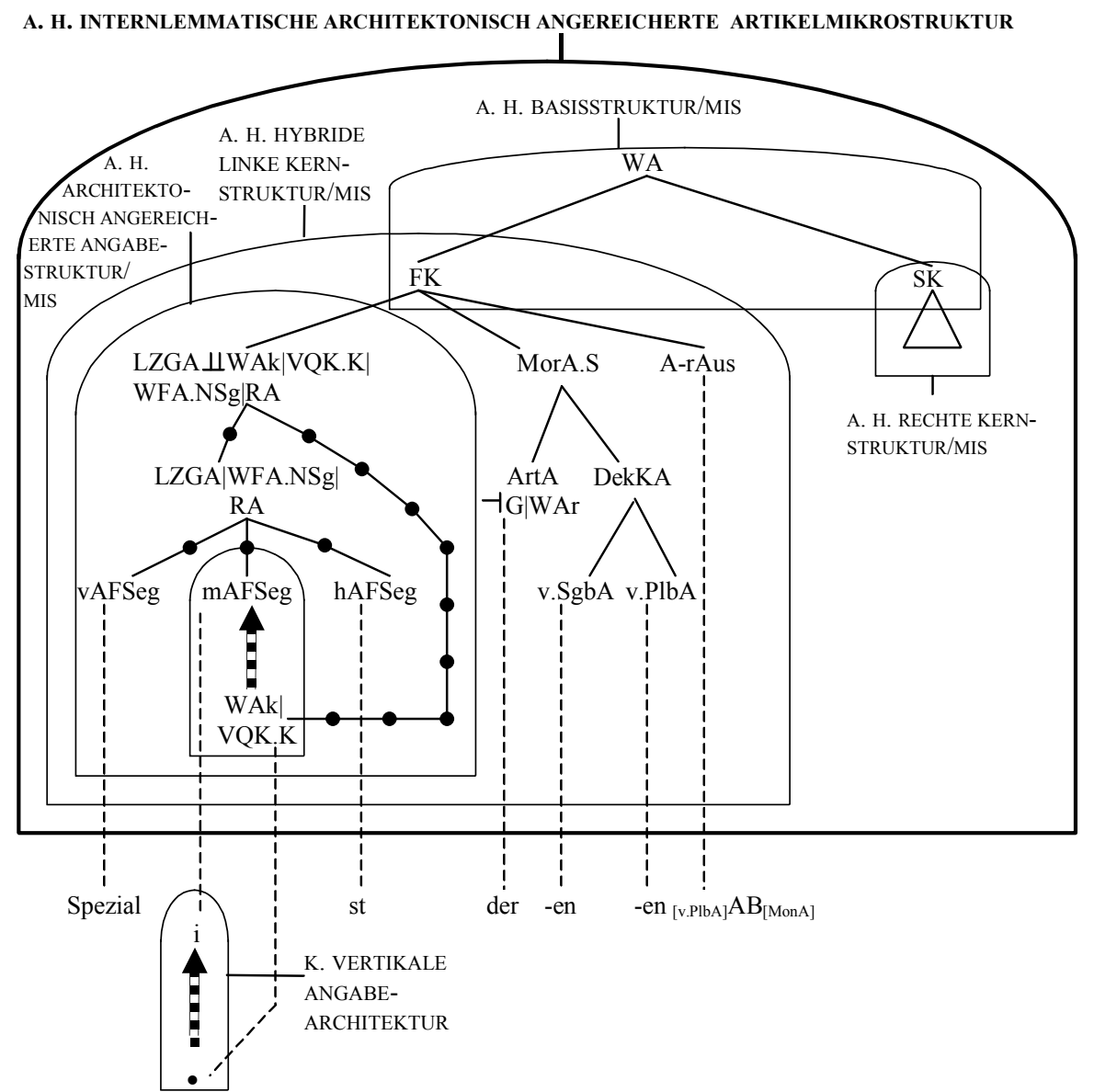

Abb. 7: Nichtvollständig ausgeführter, einfach kommentierter und mit architektonischen Komponenten angereicherter Strukturgraph zur abstrakten (und isomorphen konkreten) hierarchischen internlemmatisch architektonisch angereicherten Artikelmikrostruktur, die wa $\mathrm{a}_{3}$ in Abb. 1 aufweist

Die Angabe "er muß einen Spezialisten (Facharzt) aufsuchen" $\left(=a_{1}\right)$ aus wa 5 in Abb. 1 ist eine um ein semantisches Binnenglossat (G.s) erweiterte Kompetenzbeispielangabe; sie erhält den Angabenamen $\mathrm{a}_{1}$; so dass gilt $\mathrm{a}_{1} \in \mathrm{KBei}(\mathrm{G} . \mathrm{s}) A$. Die Angabe $a_{1}$ gehört zu den elementaren Angaben, da $a_{1}$ funktional-positional nicht segmentierbar ist; $a_{1}$ weist mithin keine (konkrete und abstrakte) Angabekonstituenten- oder (konkrete und abstrakte) Angabemikrostrukturen als deren Teilstrukturen auf. Dennoch weist die Angabe a eine Struktur auf, die ein Benutzer, der anhand dieser Angabe eine Information gewinnen möchte, auch intuitiv erkennen muss. Diese konkrete $(k)$ hierarchische glossatbedingte 
(g) Angabestruktur (AnS) ist wie folgt erhältlich: Man bildet zunächst eine elementenheterogene Trägermenge - sie heiße $M_{\mathrm{gAnS}}^{\mathrm{k}}\left(\mathrm{a}_{1}\right)$ - die als Elemente diejenigen Segmente enthält, die durch eine Anwendung der Methode der nichtfunktionalen-positionalen Segmentationen auf $\mathrm{a}_{1}$ erhältlich sind. $\mathrm{M}_{\mathrm{gAnS}}^{\mathrm{k}}\left(\mathrm{a}_{1}\right)$ kann wie folgt angegeben werden:

$$
\mathrm{M}_{\mathrm{gAnS}}^{\mathrm{k}}\left(\mathrm{a}_{1}\right)=\left\{\mathrm{b}_{1}, \mathrm{c}_{1}, \mathrm{~d}_{1}, \mathrm{e}_{1}, \mathrm{f}_{1}, \mathrm{a}_{1}\right\} \text {. }
$$

Die Elemente der Trägermenge sind wie folgt erklärt:

$$
\begin{aligned}
& \mathrm{b}_{1}=\text { er muß einen Spezialisten; } \mathrm{b}_{1} \in \mathrm{vASeg} \text { (= vorderes Angabesegment). } \\
& \mathrm{c}_{1}=\left(; \mathrm{c}_{1} \in v Z O Z\right. \text { (= vorderes Zusammenordnungszeichen). } \\
& \mathrm{d}_{1}=\text { Facharzt; } \mathrm{d}_{1} \in \text { G.s (= semantisches Binnenglossat). } \\
& \left.\mathrm{e}_{1}=\right) ; \mathrm{e}_{1} \in h Z O Z ;(=\text { hinteres Zusammenordnungszeichen). } \\
& \mathrm{f}_{1}=\text { aufsuchen; } \mathrm{f}_{1} \in \text { hASeg (= hinteres Angabesegment). }
\end{aligned}
$$

Nach der Bildung der Trägermenge $\mathrm{M}_{\mathrm{gAnS}}^{\mathrm{k}}\left(\mathrm{a}_{1}\right)$ definiert man auf dieser zwei strukturprägende Relationen, und zwar eine vom Typ der Präzedenzrelation - sie heiße $\mathrm{R}_{\mathrm{p}}^{\mathrm{k}}\left(\mathrm{a}_{1}\right)$ - mit dem Relationsterm $x$ geht voraus $y$ (mit ", $x^{\prime \prime}$ und ", $y^{\prime \prime}$ als Variablen für Textsegmente), sowie eine vom Typ der partitiven Relation sie heiße $\mathrm{R}_{\text {part }}^{\mathrm{k}}\left(\mathrm{a}_{1}\right)$ - mit dem Relationsterm $x$ ist ein Teil von $y$ (mit ", $x^{\prime \prime}$ als Variable für Textsegmente und ", $y$ " als Variable für binnenerweiterte Angaben). Es gelten: $\mathrm{R}_{\mathrm{p}}^{\mathrm{k}}\left(\mathrm{a}_{1}\right) \subseteq \mathrm{M}_{\mathrm{gAnS}}^{\mathrm{k}}\left(\mathrm{a}_{1}\right) \times \mathrm{M}_{\mathrm{gAnS}}^{\mathrm{k}}\left(\mathrm{a}_{1}\right) ; \mathrm{R}_{\mathrm{part}}^{\mathrm{k}}\left(\mathrm{a}_{1}\right) \subseteq \mathrm{M}_{\mathrm{gAnS}}^{\mathrm{k}}\left(\mathrm{a}_{1}\right) \times \mathrm{M}_{\mathrm{gAnS}}^{\mathrm{k}}\left(\mathrm{a}_{1}\right)$; $\mathrm{R}_{\mathrm{p}}^{\mathrm{k}}\left(\mathrm{a}_{1}\right) \cap \mathrm{R}_{\mathrm{part}}^{\mathrm{k}}\left(\mathrm{a}_{1}\right)=\varnothing$. Die konkrete hierarchische glossatbedingte Angabestruktur von $\mathrm{a}_{1}$, die sich nach der Ausführung der genannten Prozeduren ergibt, ist in Abb. 8 (1) dargestellt. Definiert man auf der Trägermenge für die zugehörige abstrakte (a) hierarchische glossatbedingte (g) Angabestruktur (AnS)

(1)

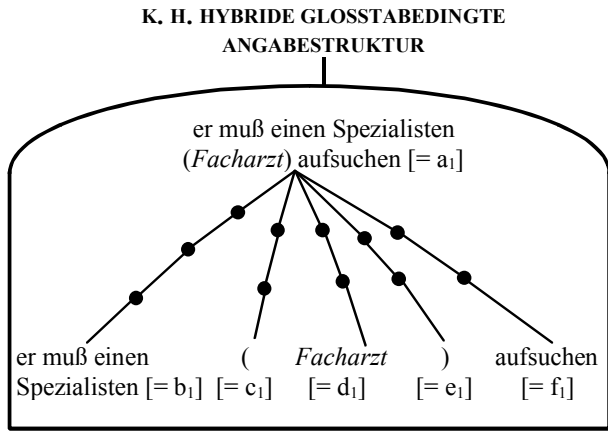

(2)

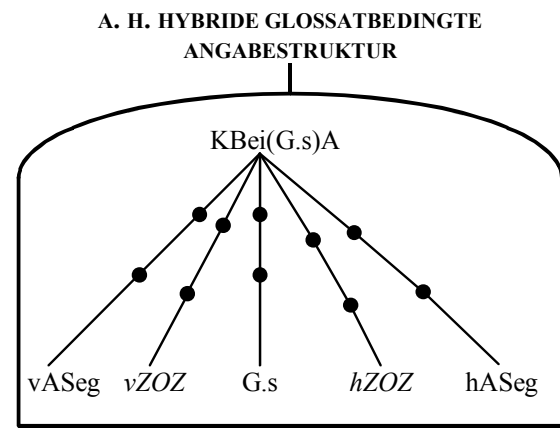

Abb. 8: Erweitert kommentierter Baumgraph zur konkreten (1) und einfach kommentierter Baumgraph zur isomorphen abstrakten (2) hierarchischen hybriden glossatbedingten Angabestruktur, die die binnenerweiterte Kompetenzbeispielangabe aus wa $\mathrm{a}_{5}$ in Abb. 1 aufweist 
- sie heiße $\mathrm{M}_{\mathrm{gAnS}}^{\mathrm{a}}\left(\mathrm{a}_{1}\right)-\left(\mathrm{M}_{\mathrm{gAnS}}^{\mathrm{a}}\left(\mathrm{a}_{1}\right)=\left\{\mathrm{M}_{\mathrm{gAnS}}^{\mathrm{a}}\left(\mathrm{a}^{1}\right)=\{\mathrm{vASeg}, v Z o Z\right.\right.$, G.s, $h Z o Z$, hASeg, KBei(G.s)A\}) zwei Relationen $\mathrm{R}_{\mathrm{p}}^{\mathrm{a}}\left(\mathrm{a}_{1}\right)$ und $\mathrm{R}_{\mathrm{part}}^{\mathrm{a}}\left(\mathrm{a}_{1}\right)$, die den auf $\mathrm{M}_{\mathrm{gAnS}}^{\mathrm{k}}\left(\mathrm{a}_{1}\right)$ definierten entsprechen, dann erhält man die abstrakte hierarchische glossatbedingte Angabestruktur von $\mathrm{a}_{1}$, die in Abb. 8 dargestellt ist.

Wie die beiden Baumgraphen in Abb. 5, so lassen sich auch die beiden Baumgraphen in Abb. $8 \mathrm{zu}$ einem Strukturgraphen zusammenfassen; dieser findet sich in Abb. 9.

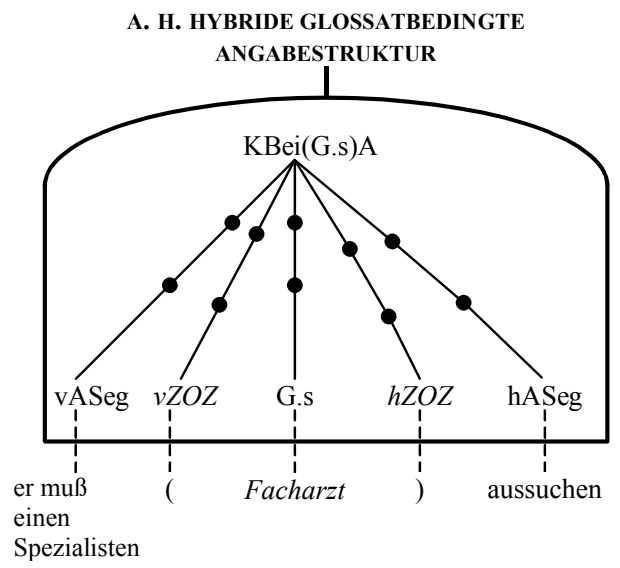

Abb. 9: Einfach kommentierter Strukturgraph zur abstrakten (und isomorphen konkreten) hierarchischen hybriden glossatbedingten Angabestruktur, die die binnenerweiterte Kompetenzbeispielangabe in wa5 in Abb. 1 aufweist

Wie aus den Abb. 8 und 9 und aus den Textzeilen zur Bildung von glossatbedingten Angabestrukturen hervorgeht, werden bei diesen Strukturen die nichttypographischen Angabestrukturanzeiger, die von nichttypographischen Angabemikrostrukturanzeigern unterschieden werden müssen (vgl. WLWF-1 2010) berücksichtigt. Dies bedeutet, dass glossatbedingte Angabestrukturen nicht als Teilstrukturen von hierarchischen Artikelmikrostrukturen auftreten, da in letzteren die nichttypographischen Strukturanzeiger keine Berücksichtigung finden. Vielmehr treten hierarchische glossatbedingte Angabestrukturen als Teilstrukturen von hierarchischen Artikelkonstituentenstrukturen auf, bei denen die nichttypographischen Strukturanzeiger als Textkonstituenten berücksichtigt werden (vgl. Abb. 14). Im Folgenden wird die in Abb. 9 in konkreter und abstrakter Ausprägung dargestellte hierarchische glossatbedingte Angabestruktur in den semantischen Kommentar der Artikelkonstituentenstruktur von wa $\mathrm{a}_{5}$ in Abb. 1 strukturell integriert. Das Ergebnis dieser Operation findet sich in Abb. 10.

Will man die hierarchische Struktur von binnenerweiterten Angaben bei der Darstellung von hybriden Artikelmikrostrukturen explizit berücksichtigen, benötigt man hierarchische Angabestrukturen, in denen die nichttypographischen Angabestrukturanzeiger nicht berücksichtigt werden. Solche Angabe- 
strukturen heißen (nach Wiegand 2007: 201f u. 2009a: 80f) hierarchische hybride minimierte glossatbedingte Angabestrukturen. Sie sind auf zweierlei Weise erhältlich. Verfügt man bereits - wie hier - über glossatbedingte Angabestrukturen, dann kann man die Trägermengen um die entsprechenden Elemente verringern. Demgemäß müssen erstens die Trägermengen für die konkreten hierarchischen glossatbedingten Angabestrukturen um die konkreten Elemente verringert werden; die Trägermengen für die abstrakten hierarchischen glossatbedingten Angabestrukturen müssen entsprechend um die Elemente verringert werden, die Klassen von nichttypographischen Strukturanzeigern sind.

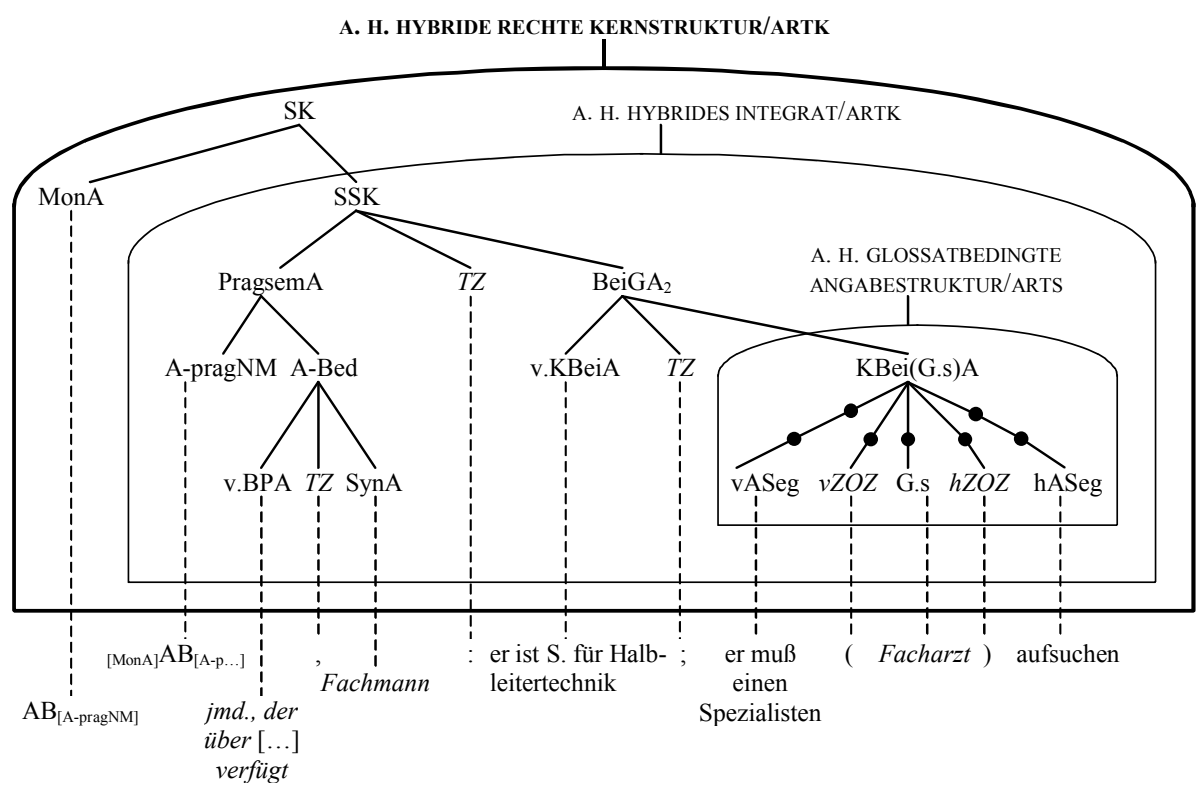

Abb. 10: Einfach kommentierter Strukturgraph zur abstrakten (und isomorphen konkreten) hierarchischen hybriden Kernstruktur der hybriden Artikelkonstituentenstruktur, die wa $\mathrm{w}_{5}$ in Abb. 1 aufweist

Abkürzungen: BeiGA $_{2}=$ Beispielgruppenangabe, die aus zwei Beispielangaben besteht; $T Z=$ Trennzeichen; $v Z O Z$ = vorderes Angabesegment; G.s = semantisches Binnenglossat; $h \mathrm{ZOZ}=$ hinteres Zusammenordnungszeichen; hASeg = hinteres Angabesegment; ARTK = ARTIKELKONSTITUENTENSTRUKTUR; KERNSTRUKTUR/ARTK ist zu lesen wie Kernstruktur als Substruktur der Artikelkonstituentenstruktur

Zweitens müssen die auf den beiden Trägermengen definierten Relationen auf die verringerte Trägermenge eingeschränkt werden, so dass alle 2-Tupel getilgt werden, in denen als Koordinate entweder (bei den konkreten Strukturen) ein nichttypographischer Strukturanzeiger auftritt, oder (bei den abstrakten Strukturen) eine Klasse von nichttypographischen Strukturanzeigern. Bei Angabe $\mathrm{a}_{1}=$,er muß einen Spezialisten (Facharzt) aufsuchen“ aus was in Abb. 1 ergibt sich dann das Folgende: 
(1) Die Trägermenge für die konkrete Struktur, nämlich $\mathrm{M}_{\mathrm{gAnS}}^{\mathrm{k}}\left(\mathrm{a}_{1}\right)$, wird um die Elemente $c_{1}$ und $\mathrm{e}_{1}$ (vgl. Abb. 8 (1)) vermindert, so dass sich die verminderte $(v)$ Trägermenge $v^{\mathrm{g}} \mathrm{gAnS}^{\mathrm{k}}\left(\mathrm{a}_{1}\right)=\left\{\mathrm{b}_{1}, \mathrm{~d}_{1}, \mathrm{f}_{1} \mathrm{a}_{1}\right\}$ ergibt. Die Relation $\mathrm{R}_{\mathrm{p}}^{\mathrm{k}}\left(\mathrm{a}_{1}\right)$ wird so eingeschränkt, dass folgende ihrer 2-Tupel, die $\mathrm{c}_{1}$ oder $\mathrm{e}_{1}$ als Koordinaten aufweisen, eliminiert werden: $\left\langle\mathrm{b}_{1}, \mathrm{c}_{1}\right\rangle,\left\langle\mathrm{b}_{1}, \mathrm{e}_{1}\right\rangle$, $\left\langle\mathrm{c}_{1}, \mathrm{~d}_{1}\right\rangle,\left\langle\mathrm{c}_{1}, \mathrm{e}_{1}\right\rangle,\left\langle\mathrm{c}_{1}, \mathrm{f}_{1}\right\rangle,\left\langle\mathrm{d}_{1}, \mathrm{e}_{1}\right\rangle$, und $\left\langle\mathrm{e}_{1}, \mathrm{f}_{1}\right\rangle$. Die eingeschränkte (e) Relation $\mathrm{eR}_{\mathrm{p}}^{\mathrm{k}}\left(\mathrm{a}_{1}\right)$ kann dann wie folgt angegeben werden: $\mathrm{eR}_{\mathrm{p}}^{\mathrm{k}}\left(\mathrm{wa}_{1}\right)=$ $\left.\left.\left\{<b_{1}, d_{1}\right\rangle,<b_{1}, f_{1}\right\rangle,<d_{1}, f_{1}>\right\}$ (vgl. Abb. 11). Die Relation $R_{\text {part }}^{k}\left(a_{1}\right)$ wird ebenfalls eingeschränkt, und zwar so, dass folgende ihrer 2-Tupel, die $c_{1}$ oder $\mathrm{e}_{1}$ als Koordinaten aufweisen, eliminiert werden: $\left\langle\mathrm{c}_{1}, \mathrm{a}_{1}\right\rangle,\left\langle\mathrm{e}_{1}, \mathrm{a}_{1}\right\rangle$. Die eingeschränkte (e) Relation kann dann wie folgt angegeben werden: $e R_{\text {part }}^{\mathrm{k}}\left(\mathrm{a}_{1}\right)=\left\{\left\langle\mathrm{b}_{1}, \mathrm{a}_{1}\right\rangle,\left\langle\mathrm{d}_{1}, \mathrm{a}_{1}\right\rangle,\left\langle\mathrm{f}_{1}, \mathrm{a}_{1}\right\rangle\right\}$.

(2) Die Trägermenge für die abstrakte (a) Struktur, nämlich $\mathrm{M}_{\mathrm{gAnS}}^{\mathrm{a}}\left(\mathrm{a}_{1}\right)$, wird um die Elemente $v Z O Z$ und $h Z O Z$ vermindert, so dass sich die verminderte (v) Trägermenge $v_{\text {gAnS }}^{\mathrm{a}}\left(\mathrm{a}_{1}\right)=\{\mathrm{vASeg}$, G.s, hASeg $\}$ ergibt. Die Relation $\mathrm{R}_{\mathrm{p}}^{\mathrm{a}}\left(\mathrm{a}_{1}\right)$ wird analog $\mathrm{zu} \mathrm{R}_{\mathrm{p}}^{\mathrm{k}}\left(\mathrm{a}_{1}\right)$ eingeschränkt und kann demgemäß wie folgt angegeben werden: $\mathrm{eR}_{\mathrm{p}}^{\mathrm{a}}\left(\mathrm{a}_{1}\right)=\{\langle\mathrm{vASeg}, \mathrm{G} . \mathrm{s}\rangle,\langle\mathrm{vASeg}, \mathrm{hASeg}\rangle$, $<$ G.s, hASeg $>$ \}. Schließlich wird noch $\mathrm{R}_{\text {part }}^{\mathrm{a}}\left(\mathrm{a}_{1}\right)$ analog $\mathrm{zu} \mathrm{R}_{\text {part }}^{\mathrm{k}}\left(\mathrm{a}_{1}\right)$ eingeschränkt und kann demgemäß wie folgt angegeben werden: $\mathrm{eR}_{\text {part }}^{\mathrm{k}}\left(\mathrm{a}_{1}\right)=$ $\{<$ vASeg, KBei(G.s)A $>,<$ G.s, KBei(G.s)A $>,<$ hASeg, KBei(G.s)A $>\}$.

Nach Ausführung der skizzierten Operationen ergeben sich die konkrete hierarchische minimierte glossatbedingte Angabestruktur sowie die zugehörige isomorphe abstrakte hierarchische minimierte glossatbedingte Angabestruktur, die $a_{1}$, die um eine semantisches Binnenglossat erweiterte Kompetenzbeispielangabe "er muß einen Spezialisten (Facharzt) aufsuchen" aus was in Abb. 1 aufweist; die beiden minimierten Strukturen sind mit dem Strukturgraphen in Abb. 11 dargestellt.

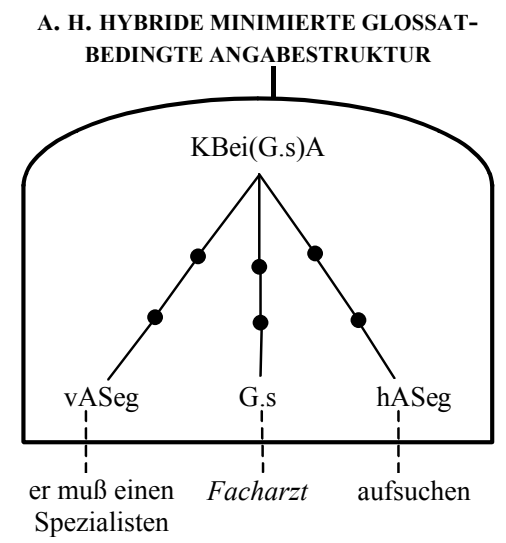

Abb. 11: Einfach kommentierter Strukturgraph zur konkreten (und isomorphen abstrakten) minimierten glossatbedingten Angabestruktur, die wa 5 in Abb. 1 aufweist 
Der skizzierte Übergang von hierarchischen glossatbedingten zu hierarchischen minimierten glossatbedingten Angabestrukturen lässt sich auch auf der Ebene der formalen Strukturdarstellung durchführen, indem man die Kanten, die $z u$ den Knoten führen, die nichttypographische Angabestrukturanzeiger repräsentieren, sowie die zugehörigen Knotenetikette tilgt und die einfache Kommentierung sinngemäß ändert.

Wir verfügen nun über alle Substrukturen, um die konkrete (und isomorphe abstrakte) hierarchische internlemmatisch architektonisch angereicherte Artikelmikrostruktur mit minimierter glossatbedingter Teilstruktur, die wa $\mathrm{w}_{5}$ in Abb. 1 aufweist, in Abb. 12 darstellen zu können.

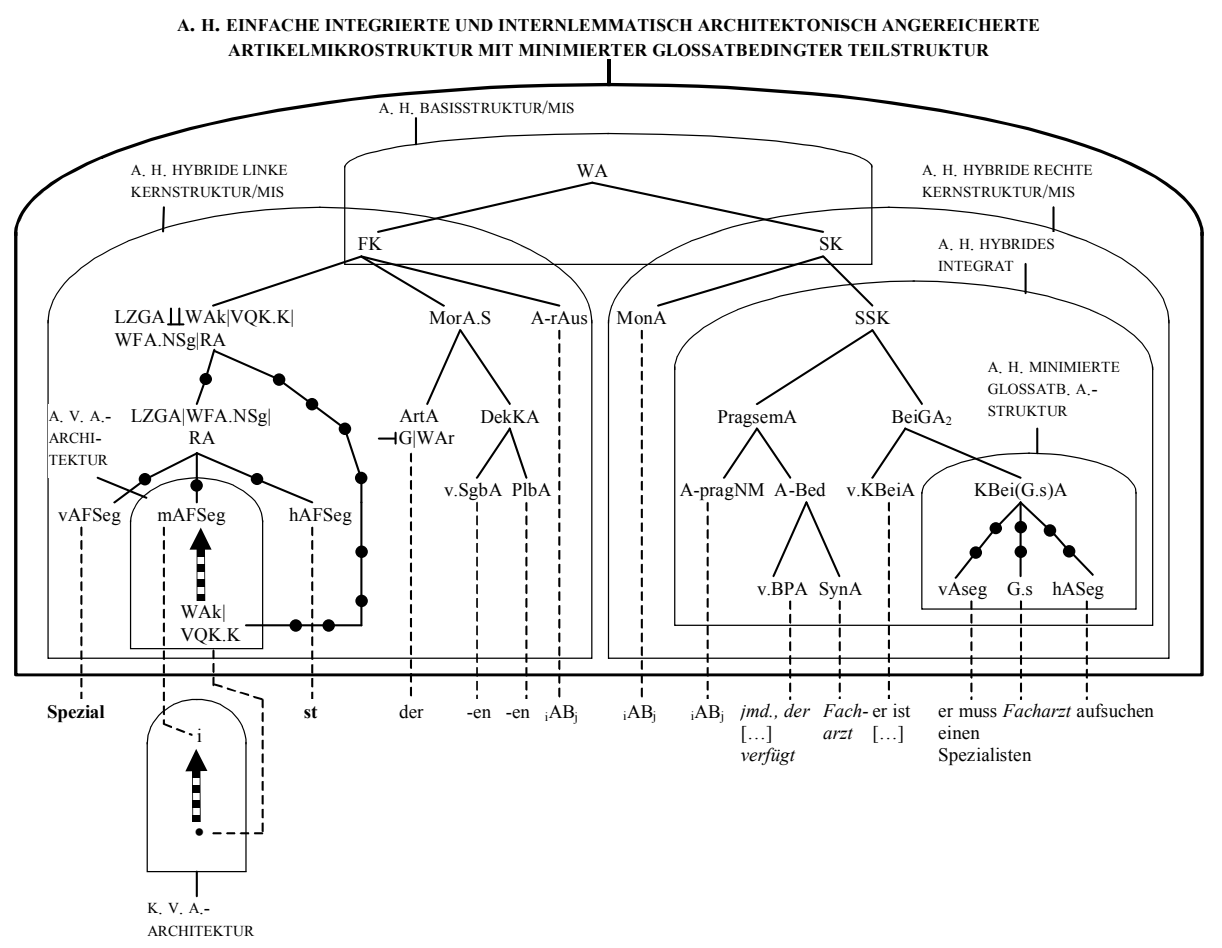

Abb. 12: Einfach kommentierter du mit architektonischen Komponenten angereicherter Strukturgraph zur abstrakten (und isomorphen konkreten) hierarchischen einfachen integrierten und internlemmatisch architektonisch angereicherten Artikelmikrostruktur mit minimierter glossatbedingter Teilstruktur

Abkürzungen: $\mathrm{vAFSeg}=$ vorderes Angabeformsegment; $\mathrm{mAFSeg}=$ mittleres Angabeformsegment; hAFSeg = hinteres Angabeformsegment; A-rAus = Angabe zur regelmäßigen Aussprache; MonA = Monosemieangabe; A-pragNM = Angabe zur pragmatischen Nullmarkierung; A-Bed = Angabe zur Bedeutung; v.BPA = verdichtete Bedeutungsparaphrasenangabe; BeiGA $_{2}=$ Beispielgruppenangabe, die aus zwei Beispielangaben (unterschiedlichen Typs) besteht; v.KBeiA = verdichtete Kompetenzbeispielangabe; KBei(G.s)A = um ein 


\begin{abstract}
semantisches Binnenglossat erweitere Kompetenzbeispielangabe; vASeg = vorderes Angabesegment; G.s = semantisches Binnenglossat; hASeg = hinteres Angabesegment; ${ }_{i} \mathrm{AB}_{\mathrm{j}}=$ Angabeblank mit Nachbarschaftsvariablen ohne Belegung. A.V.A.-ARCHITEKTUR = ABSTRAKT VERTIKALE ANGABEARCHITEKTUR; K.V.A.-ARCHITEKTUR = KONKRETE VERTIKALE ANGABEARCHITEKTUR
\end{abstract}

Im Folgenden betrachten wir wa ${ }_{6}$ in Abb. 1. Der Artikel weist eine konkrete (und isomorphe abstrakte) hierarchische rechtserweiterte integrierte hybride Artikelmikrostruktur mit minimierter doppelglossatbedingter Teilstruktur auf. Das Doppelglossat findet sich in der verdichteten Kompetenzbeispielangabe „das hat viel S. (Mühe, Anstrengung) gekostet". Ein Doppelglossat ist ein Glossat, das aus zwei aufeinanderfolgenden gleichadressierten Binnenglossaten besteht, zwischen denen ein Trennzeichen steht. Die Glossate sind hier nicht nur gleichadressiert, sondern auch klassengleich: Es handelt sich hier um zwei semantische Binnenglossate. Für Doppelglossate mit zwei klassengleichen Glossaten gibt es zwei Analysemöglichkeiten, zu denen zwei unterschiedliche Strukturkonzeptionen gehören.

(1) Die erste Analysemöglichkeit wurde in Wiegand (2009a: 79f) vorgeführt; sie basiert darauf, dass das Konzept der homosegmentären Textsegmente nur für klassengleiche Angaben gelten soll. Nach diesem Strukturkonzept ist z.B. die Angabe "Knabe, Junge" in wa7 in Abb. 14 eine (nichtelementare) zweifach homosegmentäre Synonymenangabe $\left(S_{y n} A^{2}\right)$, die in zwei (elementare) Synonymangaben (SynA) funktional-positional segmentierbar ist (vgl. Wiegand 2005: 229f). Das semantische Doppelglossat "Mühe, Anstrengung" in wa6 wird dann nicht als homosegmentäres Textsegment analysiert. Als Grund dafür kann angegeben werden, dass alle binnenerweiterungsbedingten hierarchischen Angabestrukturen als flache hierarchische Strukturen (i.S.v. Wiegand 2009: 254f) zu konzipieren sind. Das sind - um es einfach auszudrücken — solche textuellen Strukturen, bei deren Darstellung mittels eines geordneten Baumgraphen außer dem Wurzelknoten nur terminale Knoten auftreten, wie z.B. in Abb. 8, 9 und 11. Diese Strukturkonzeption ist vertretbar, wenn in einem Wörterbuch nur elementare Binnenglossate und nichtelementare Doppelglossate auftreten, die aus zwei klassengleichen Binnenglossaten bestehen. Treten nichtelementare Binnenglossate auf, die $\mathrm{zu}$ anderen Typen gehören, wie in wa 8 in Abb. 15, ist die zweite Strukturkonzeption vorzuziehen.

(2) Die zweite Analysemöglichkeit ergibt sich, wenn man das Konzept der homosegmentären Textsegmente auch für Doppelglossate gelten lässt und damit tiefe hierarchische Strukturen bei den binnenerweiterungsbedingten Angabestrukturen zulässt, also solche Strukturen, bei deren Darstellung mittels eines geordneten Baumgraphen mindestens ein Kantenzug auftritt, so dass die Höhe des Baumgraphen mindestens den 
Wert 2 hat. In Abb. 13 ist die konkrete (und isomorphe abstrakte) hierarchische minimierte doppelglossatbedingte Angabestruktur der um ein semantisches Doppelglossat binnenerweiterten verdichteten Kompetenzbeispielangabe des metaphorischen Wortgebrauchs "das hat viel S. (Mühe, Anstrengung) gekostet" aus wa 6 sowohl als flache (1) als auch als tiefe (2) Struktur dargestellt.

(2)

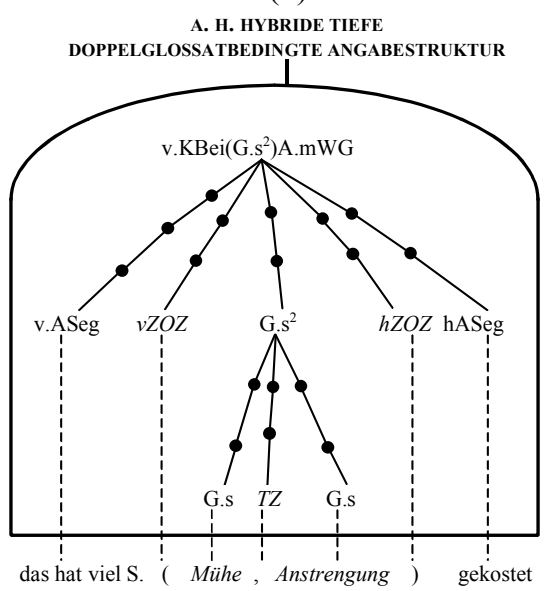

Abb. 13: Einfach kommentierte Strukturgraphen zur abstrakten (und isomorphen konkreten) hierarchischen doppelglossatbedingten Angabestruktur, konzipiert als flache Struktur (1) und als tiefe Struktur (2) Abkürzungen: DG.s = semantisches Doppelglossat; G.s ${ }^{2}=$ zweifach homosegmentäres semantisches Binnenglossat (= semantisches Doppelglossat, verstanden als homosegmentäres Textsegment); v.KBei(DG.s)A.mWG = um ein semantisches Doppelglossat binnenerweiterte verdichtete Kompetenzbeispielangabe des metaphorischen Wortgebrauchs

$\mathrm{Zu}$ dem Segmentationsprozess als ersten Prozessabschnitt des Strukturbildungsprozesses, der zu der in Abb. 13 (2) dargestellten tiefen Struktur geführt hat, sei das Folgende nachgetragen: Es gehört zu den Korrektheitsbedingungen bei der Anwendung der Methode der nichtfunktionalen-positionalen Segmentation, dass ihre Anwendung zu mindestens einem nichtfunktionalen Textsegment führt; es gehört aber nicht zu den Korrektheitsbedingungen, dass alle erhältlichen Textsegmente nichtfunktionale Textsegmente sind. Demgemäß läuft die Segmentation der um ein semantisches Doppelglossat binnenerweiterten verdichteten Kompetenzbeispielangabe des metaphorischen Wortgebrauchs (v.KBei(DG.s)A.mWG) wie folgt ab: Im ersten Schritt wird "das hat viel S. (Mühe, Anstrengung) gekostet" nichtfunktional-positional segmentiert, so dass fünf Textsegmente gegeben sind (das hat viel S. |( |Mühe, Anstrengung |) | gekostet, mit "|" als Segmentationsfugenmarkierung). Da diese fünf Textsegmente nicht das Ergebnis einer Anwendung der funktional-positionalen 
Segmentation sind, sind sie keine Textkonstituenten. Zwei Textsegmente sind nichtfunktional, nämlich "das hat viel S." $\in$ vASeg" und "gekostet" $\in$ hASeg. Der diskontinuierliche angabeinterne Strukturanzeiger, das Zusammenordnungszeichen "( )", das durch ein Zeichen-Tupel realisiert ist, ist dagegen ein funktionales Textsegment. Das Doppelglossat ist ein nichtelementares funktionales Textsegment ohne Textkonstituentenstatus, da es - wie bereits gesagt - nicht aus einer Anwendung einer der Methodenvarianten der Methode der funktional-positionalen Segmentation hervorgegangen ist. Auf das isolierte Doppelglossat "Mühe, Anstrengung“ lässt sich nun allerdings die Methode der exhaustiven funktional-positionalen Segmentation anwenden, da das Doppelglossat restfrei in drei funktionale Textsegmente segmentiert werden kann. Daher ergibt sich nun die Frage, ob diese drei Textsegmente Textkonstituentenstatus aufweisen oder nicht. Diese Frage lässt sich durch Rückgriff auf die zentrale Korrektheitsbedingung für die Anwendung der Methode der funktinal-positionalen Segmentation beantworten, die lautet: „Durch die Anwendung der Methode der funktional-positionalen Methode auf ein Textsegment TS entstehen ausschließlich und mindestens zwei funktionale Textsegmente, die genau dann Textkonstituentenstatus haben, wenn TS eine Textkonstituente ist. Ist TS ein Textsegment ohne Textkonstituentenstatus, dann sind die durch eine Anwendung der funktional-positionalen Methode erhältlichen funktionalen Textsegmente hybride Textkonstituenten" (vgl. Wiegand 2010). Da das Doppelglossat keine Textkonstituente ist, aber funktional positional segmentierbar, sind "Mühe“, das Komma und "Anstrengung" hybride Textkonstituenten. Damit ist der erste Typ der hybriden textuellen Einheiten identifiziert (vgl. 7).

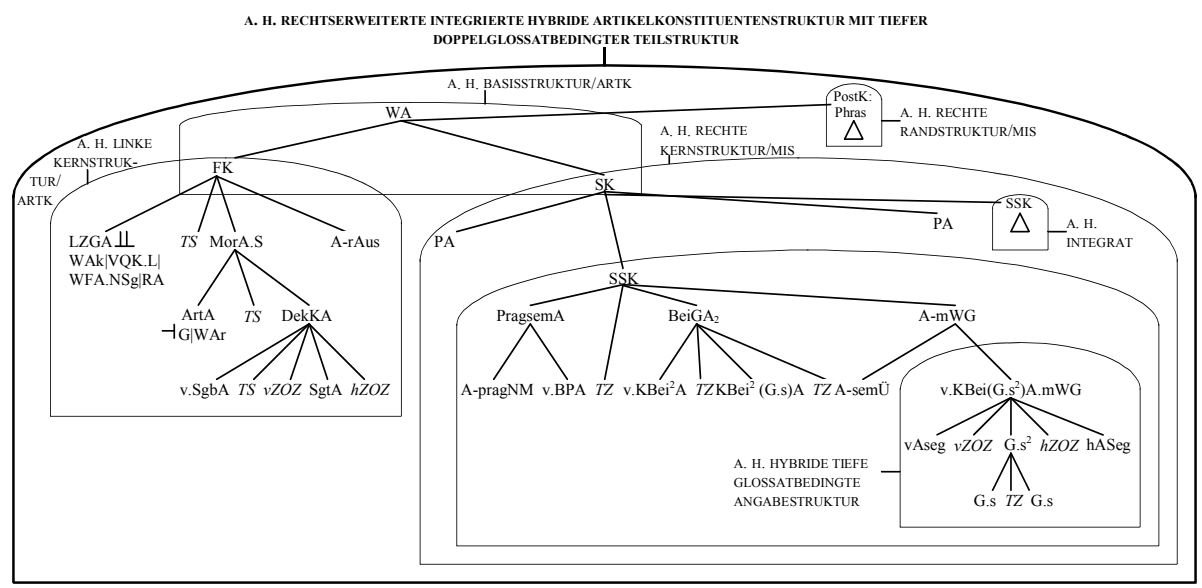

Abb. 14: Nichtvollständig ausgeführter einfach kommentierter Strukturgraph zur abstrakten hierarchischen rechtserweiterten integrierten hybriden Artikelkonstituentenstruktur mit tiefer doppelglossatbedingter Teilstruktur, die wa ${ }_{6}$ in Abb. 1 aufweist 
Abkürzungen: SgtA = Singularetantumangabe; v.KBei²A = verdichtete Kompetenzbeispielangabe, anhand derer zwei Beispiele erschlossen werden können; A.mWG = Angabe des metaphorischen Wortgebrauchs; A-semÜ = Angabe zum semantischen Übergang; v.KBeiA(G.s²)A.mWG = verdichtete Kompetenzbeispielangabe des metaphorischen Wortgebrauchs, binnenerweitert um ein zweifach homosegmentäres Glossat (= Doppelglossat)

Im Folgenden wird die in Abb. 13 (2) dargestellte abstrakte hierarchische tiefe doppelglossatbedingte Angabestruktur in die Artikelkonstituentenstruktur von wa $_{6}$ in Abb. 1 integriert. Der Strukturbildungsprozess wird nicht beschrieben, sondern es wird nur sein Ergebnis in Abb. 14 partiell dargestellt.

Zum Abschluss dieses Abschnittes findet sich in Abb. 15, um einen partiellen Überblick zu ermöglichen, ein Ausschnitt aus einer Typologie hierarchischer hybrider textueller Wörterbuchstrukturen, der auch erkennen lässt, welche Typen in diesem Beitrag nicht behandelt werden konnten und der in Abschnitt 5 ergänzt wird (vgl. Abb. 20).

$\mathrm{Zu}$ den Termini für hierarchische hybride Artikelkonstituenten- und für hierarchische hybride Artikelmikrostrukturen ist zu bemerken: Alle architektonisch angereicherten Textkonstituentenstrukturen sind hybrid, so dass in einem Terminus, in dem das komplexe Prädikat textarchitektonisch angereicherte auftritt, das Prädikat hybrid überflüssig ist.

\section{Hybride artikelinterne Textkonstituentenstrukturen II: Angabekon- stituenten- und Angabemikrostrukturen}

Hybride konstituentenlose Angabestrukturen sind bereits bekannt. Die in Abb. 5 (1) u. (2), Abb. 6, Abb. 8 (1) u. (2), Abb. 9 und Abb. 11 dargestellten Strukturen von elementaren durch funktionale Angabezusätze erweiterten Angaben gehören jeweils zu Untertypen des Typs der hybriden konstituentenlosen Angabestruktur (vgl. Abb. 20). Während hybride konstituentenlose Angabestrukturen stets zu elementaren oben oder unten oder binnenerweiterten Angaben gehören, sind hybride Angabekonstituentenstrukturen und deren Teilstrukturen, die hybriden Angabenmikrostrukturen, stets Strukturen von nichtelementaren Angaben. Auch Beispiele für diese Strukturen, nämlich bestimmte Kommentarstrukturen, sind bereits bekannt. Der semantisch Kommentar von wa $_{5}$ in Abb. 1 ist (als unmittelbare Textkonstituente des Wörterbuchartikels was) eine nichtelementare Angabe mit Kommentarstatus. Die in Abb. 10 dargestellte konkrete hierarchische hybride rechte Kernstruktur ist als Teilstruktur der hierarchischen hybriden Artikelkonstituentenstruktur eine konkrete hybride Angabekonstituentenstruktur und gehört daher auch zum Typ der konkreten hierarchischen hybriden Angabekonstituentenstruktur mit flacher doppelglossatbedingter Teilstruktur (vgl. Abb. 20). Entsprechendes gilt für die zugehörigen abstrakten Strukturen. - Die in Abb. 12 dargestellte abstrakte hierarchische hybride linke Kernstruktur gehört als Teilstruktur der abstrakten hierarchischen hybriden Artikelmikrostruktur zu den abstrakten hierarchischen 


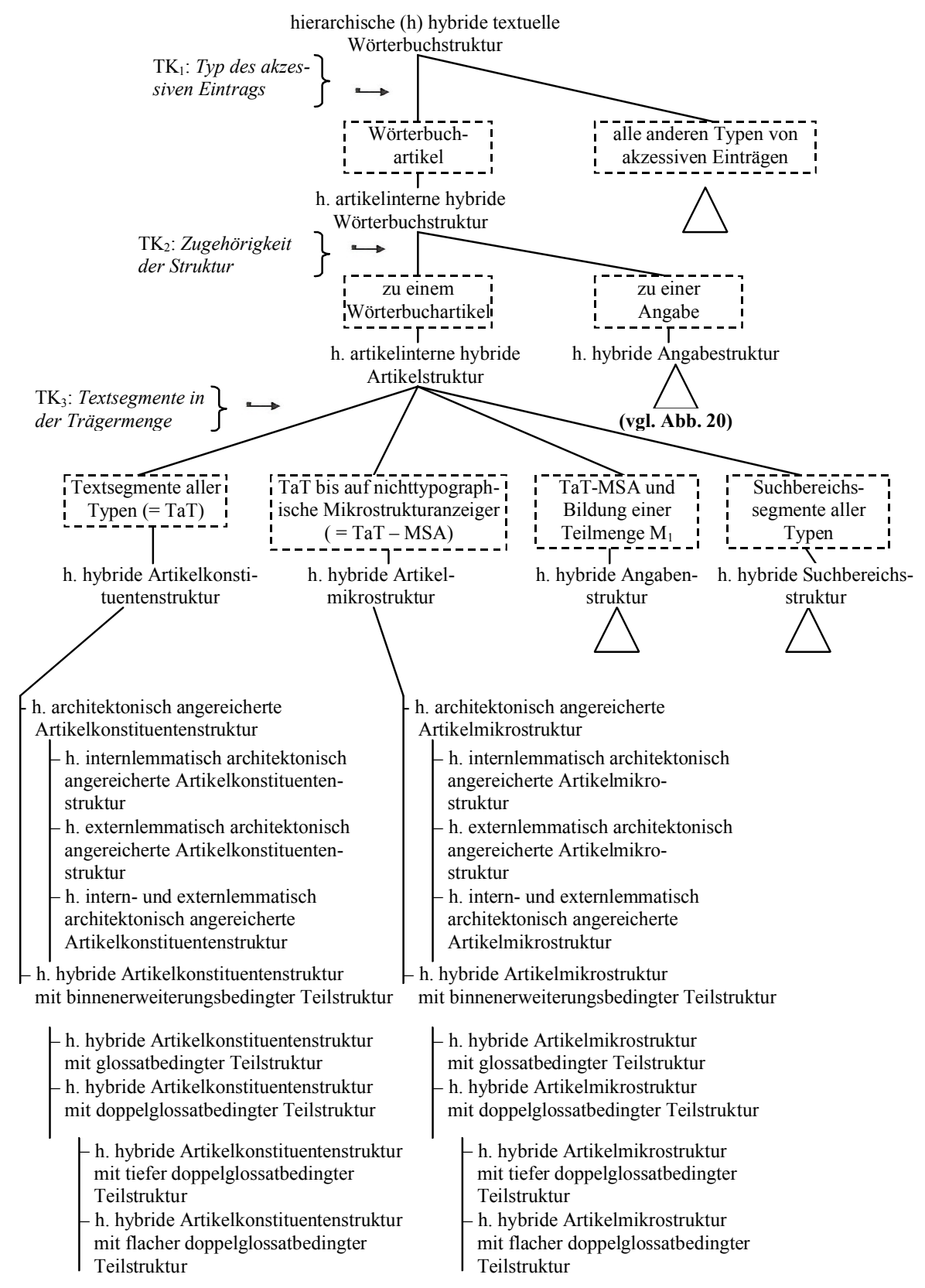

Abb. 15: Partiell erweitert kommentierter Typologiegraph zu einem Ausschnitt aus einer Typologie hierarchischer hybrider textueller Wörterbuchstrukturen

Abkürzungen: $\mathrm{TK}=$ Typologiekriterium; $\mathrm{h} .=$ hierarchisch; $\mathrm{TaT}=\mathrm{Textseg}-$ mente aller Typen; Tat-MSA = Testsegmente aller Typen minus nichttypogra- 
phische Mikrostrukturanzeiger; $\mathrm{M}_{1}=$ Teilmenge der Trägermenge, deren Elemente alle adressierten Angaben sowie alle bezugsadressentragende Angaben und damit alle Adressen sind

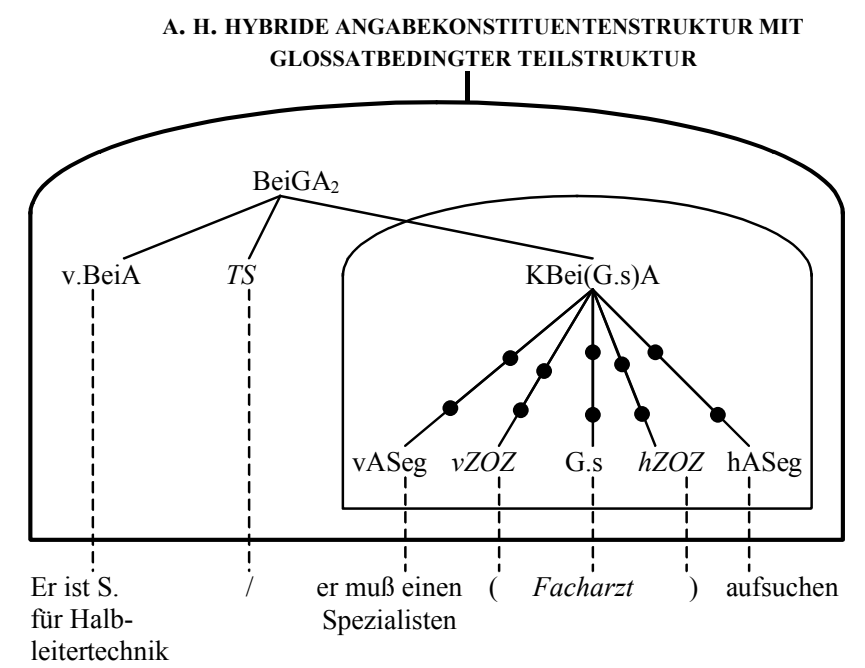

Abb. 16: Einfach kommentierter Strukturgraph zur abstrakten (und isomorphen konkreten) hierarchischen hybriden Angabekonstituentenstruktur mit glossatbedingter Teilstruktur, die die Beispielgruppenangabe $a_{5}$ aus wa in $_{5}$ Abb. 1 aufweist (vgl. Abb. 12).

hybriden Kommentarmikrostrukturen. Ordnet man sie aber in die Typologie der hierarchischen hybriden Angabestrukturen ein, in der der Kommentarstatus von Angaben keine Rolle spielt, gehört sie in ihrer konkreten und abstrakten Ausprägung zum Typ der hierarchischen internlemmatisch architektonisch angereicherten Angabemikrostruktur (vgl. Abb. 20). Entsprechendes gilt für die zugehörigen konkreten Strukturen. - Die in Abb. 12 dargestellte abstrakte hierarchische hybride rechte Kernstruktur gehört als Teilstruktur der abstrakten hierarchischen hybriden Artikelmikrostruktur zu den abstrakten hierarchischen hybriden Kommentarmikrostrukturen. Innerhalb der Typologie der hierarchischen hybriden Angabestrukturen gehört sie aber in ihrer konkreten und abstrakten Ausprägung zum Typ der hierarchischen hybriden Angabemikrostruktur mit minimierter glossatbedingter Teilstruktur (vgl. Abb. 20). Häufig gehören hierarchische hybride Angabekonstituenten- und Angabemikrostrukturen in der Typologie der Kommentarstrukturen $\mathrm{zu}$ den hierarchischen hybriden Kommentarkonstituenten - bzw. zu den Kommentarmikrostrukturen. Es gibt aber auch viele Fälle, in denen nichtelementare Angaben ohne Kommentarstatus hierarchische hybride Angabekonstituentenstrukturen und als deren Teilstrukturen hierarchische hybride Angabemikrostrukturen aufweisen. Ein Beispiel findet sich in wa in Abb. 1. Die Beispielgruppenangabe $\left(\right.$ BeiGA $\left._{2}\right)$ „er ist $\mathrm{S}$. für Halbleitertechnik; er muss einen Spezialisten (Facharzt) 
aufsuchen" ( $=\mathrm{a}_{5 \mathrm{i}} \mathrm{a}_{5} \in$ BeiGA $_{2}$ ) weist eine hierarchische hybride Angabekonstituentenstruktur mit glossatbedingter Teilstruktur auf und als deren Teilstruktur eine hierarchische hybride Angabemikrostruktur mit minimierter glossatbedingter Teilstruktur (vgl. Abb. 20). Die zuletzt genannte Angabekonstituentenstruktur ist in Abb. 16 dargestellt.

Auf der Ebene der Strukturdarstellung ist die abstrakte und isomorphe konkrete hierarchische hybride Angabemikrostruktur mit minimierter glossatbedingter Teilstruktur von $\mathrm{a}_{5}$ dadurch erhältlich, dass man erstens in Abb. 16 die Kanten mit den Endknoten TS, vZOZ und hZOZ und zweitens die zugehörigen Repräsentationen der Element-Klassen-Beziehung ( $\mathrm{x}----\mathrm{Y})$ sowie die zugehörigen Elemente in der konkreten Struktur tilgt.

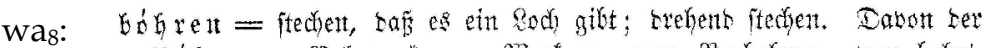

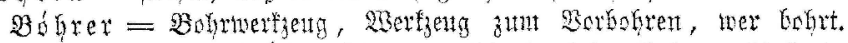

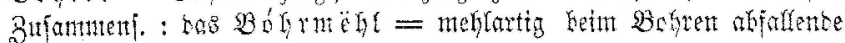
Solytheildert.

wa9: der/ Méiter \{des Meters, ritkán die Medas ter, $\mathrm{m}\}$

Der Schrank ist 2 Meter $\{S g\}$ hoch

und ein(en) Meter breit. A szekrény

2 méter magas és egy méter széles.

Meine kleine Schwester ist ein $(\underline{\mathrm{en}})$

Meter fünfzig groß. A húgom egy méter ötven magas.

Der Maurer baut eine Mauer von 30

Meter $\{S g\}$ Länge. A kömúves egy

30 méter hosszú falat épit.

wa10: klein [klain] 〈Adj.〉 1. 〈Steig. reg.〉 'in räumlicher Ausdehnung unter einem bestimmten (mittleren) Wert liegend; ANT groß $(1,2.1)$ : ein er Mann, Baum; ein es Zimmer, Haus, Format, Paket; e Hände: das Grundstück ist mir zu ; der Anzug ist, die Schuhe sind mir zu geworden; er ist $\sim e r$ als ich; ein Wort ('mit kleinen Buchstaben') schreiben; /in den kommunikativen Wendungen/ , aber oho /wird von jmdm. gesagt, der nicht groß, aber bemerkenswert energisch, leistungsfähig ist/; , aber mein ('nicht sehr groß, aber es gehört mir') /sagt jmd., wenn er betonen will, dass er mit Räumlichkeiten, Fahrzeugen od. Gegenständen zufrieden ist, weil sie ihm gehören, auch wenn sie klein sind/ 2. 〈o. Steig.; nicht bei Vb.) 'einen relativ kurzen Zeitraum umfassend' /beschränkt verbindbar/: eine e (ANT große) Pause; eine $\sim e$ Weile warten; einen $\sim e n$ Moment, bitte! 3. 〈Steig. reg.; nicht bei $\mathrm{Vb}$.〉 'aus einer relativ geringen Anzahl, Menge bestehend'; ANT groß (4) /vorw. auf Gruppen, Mengen bez./: eine e Familie, Gruppe, Herde; etw. im en Kreis besprechen; eine e Summe, Anzahl: eine e Summe Geld, Anzahl, Personen; ein es (SYN 'niedriges 2') Gehalt; 


\section{wa w11 $^{*}$ Citadelle, f. - n: eine bei einer Stadt gelegne, sie beherrschende Festung.}

Abb. 17: Wörterbuchartikel wa $a_{8}$ aus Weigand 1873 (gekürzt), wa9 aus Hollós 2001 wa 10 aus DGWDaF und wa 11 aus Sanders-WDS

In wa 8 ist "Zusammens.: das B ó h r m ë h l“ eine um „das", eine Artikelangabe, binnenerweiterte Angabe zur Komposition. Die letzte Teilangabe ist die zweifach oben erweiterte nichtlemmatische Kompositumangabe "B ó h r m ë h l“. Der erste oben erweiternde funktionale Angabezusatz ist eine Wortakzent-

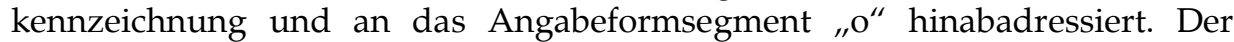
zweite oben erweiternde funktionale Angabezusatz ist eine Vokalidentifizierungskennzeichnung, mit der nach der "Vorrede“ in Weigand (1873, VII) das mit dem Angabeformsegmente genannte $e$ „nach Jacob Grimms Vorgang im

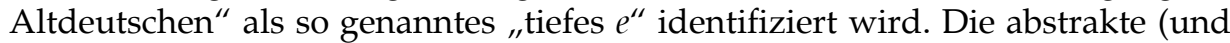
isomorphe konkrete) externlemmatisch architektonisch angereicherte Angabenmikrostrukturen sind in Abb. 18 dargestellt.

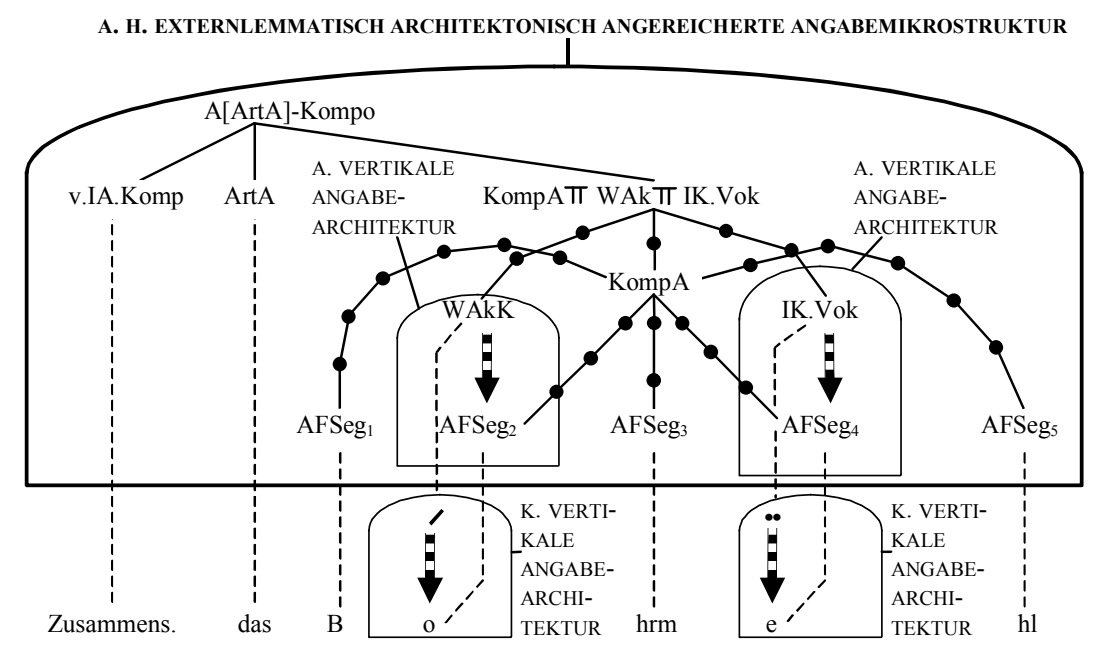

Abb. 18: Einfach kommentierter und mit architektonischen Komponenten angereicherter Strukturgraph zur (abstrakten und isomorphen konkreten) hierarchischen externlemmatisch architektonisch angereicherten Angabemikrostruktur der nichtlemmatischen um eine Artikelangabe (ArtA) binnenerweiterte Angabe zur Komposition aus wa. (die Teilstrukturen der Artikelmikrostrukturen von wa 8 sind)

Abkürzungen: A[ArtA]-Kompo = um eine Artikelangabe binnenerweiterte Angabe zur Komposition; v.IA.Komp = verdichtete Kompositumidentifizierungsangabe; KompA = Kompositumangabe; WAkK = Wortakzentkennzeichnung; IK.Vok = Vokalidentifizierungskennzeichnung; „u $\longrightarrow-\mathrm{v}^{\prime \prime}$ bedeutet

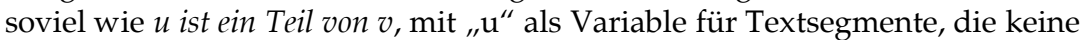
Textkonstituenten sind; "a $\rightarrow \mathrm{b}^{\prime \prime}$ bedeutet soviel wie $a$ ist oberhalb von $b$, wenn der Pfeil nach unten zeigt; ${ }_{,}, T^{\prime \prime}$ bedeutet soviel wie oben erweitert um 
Im Folgenden betrachten wir den bilingualen Wörterbuchartikel wa9, und zwar die Angabe eines äquivalenten Satzpaares „Der Maurer baut eine Mauer von 30 Meter $\{S g\}$ Länge. A kömüves egy 30 méter hosszú falt épit" (= a9; $\mathrm{a}_{9} \in \mathrm{A}$. .äSP). Die nichtelementare Angabe besteht aus einer deutschen Kompetenzbeispielangabe, auf die als zweite zielsprachliche Teilangabe eine Angabe eines äquivalenten Satzes (A.äS) folgt. Die deutsche Kompetenzbeispielangabe ist durch einen unten erweiternden funktionalen Angabezusatz, bei dem es sich um eine durch einen Unterstrich realisierte Fehlerquellenkennzeichnung (von) handelt erweitert, sowie weiterhin durch ein grammatisches Binnenglossat "Sg", bei dem es sich um eine Numeruskennzeichnung handelt. Damit weist die Angabe a9 eine hierarchische externlemmatisch achitektonisch angereicherte Angabekonstituentenstruktur mit glossatbedingter Teilstruktur auf, zu der als Teilstruktur eine hierarchische externlemmatisch architektonisch angereicherte Angabemikrostruktur mit minimierter glossatbedingter Teilstruktur gehört. Letztere ist in Abb. 19 dargestellt.

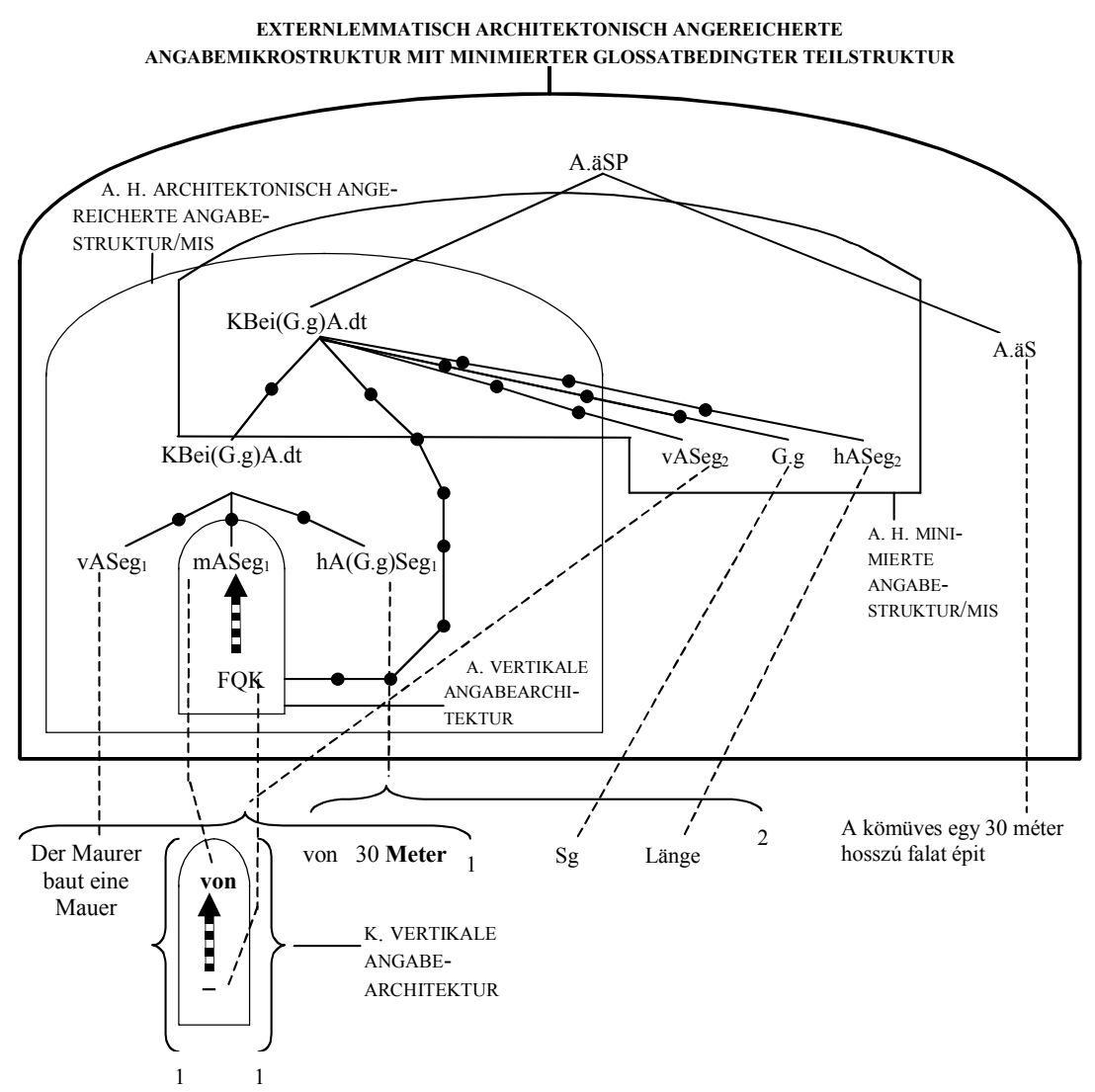

Abb. 19: Einfach kommentierter und mit architektonischen Komponenten angereicherten Strukturgraph zur abstrakten (und konkreten isomor- 
phen) hierarchischen externlemmatisch architektonisch angereicherten Angabemikrostruktur mit minimierter glossatbedingter Teilstruktur, die a9, die Angabe eines äquivalenten Satzpaares aus wa9, aufweist

Abkürzungen: A.äSP = Angabe eines äquivalenten Satzpaares; A.äS = Angabe eines äquivalenten Satzes; KBei(G.g)A.dt = deutsche (dt.) um ein grammatisches Binnenglossat (G.g) erweiterte Kompetenzbeispielangabe; FQK = Fehlerquellenkennzeichnung , $\mathrm{x} \cdots \mathrm{y}^{\prime \prime}$ bedeutet soviel wie $x$ ist unterhalb von $y$, wenn der Pfeil nach oben zeigt; Darstellungskonventionen: die mit 1 und 2 indizierten Klammern sollen helfen, die terminalen Elemente der konkreten Struktur den richtigen Klassen der abstrakten Struktur zuzuordnen

Die Typologie der hierarchischen hybriden Angabestrukturen ist reichhaltig. Einen kleinen Ausschnitt findet sich in Abb. 20.

Das mit dem Typologiegraph in Abb. 20 nur ein kleiner Ausschnitt aus einer Typologie für hierarchische hybride Angabestrukturen erfasst wird, wird sofort deutlich, wenn man bedenkt, dass hierarchische architektonisch angereicherte Angabekonstituenten- und Angabemikrostrukturen zusätzlich eine hierarchische hybride binnenerweiterungsbedingte bzw. eine hierarchische hybride minimierte binnenerweiterungsbedingte Teilstruktur aufweisen können, wie z.B. wa9 in Abb. 17 (vgl. Abb. 20).

\section{Hybride artikelinterne Textkonstituentenstrukturen III: Angaben- strukturen und exhaustive Angabenstrukturen}

Hierarchische Angabestrukturen sind - wie wir im Abschnitt 5 gesehen haben - Strukturen von erweiterten elementaren oder nichtelementaren Angaben. Hierarchische Angabenstrukturen sind dagegen textuelle Strukturen von ganzen Wörterbuchartikeln und seltener solche von anderen akzessiven Einträgen, die hier nicht berücksichtigt werden. Ein hierarchische hybride Angabenstruktur eines Wörterbuchartikels ist die um die nichthierarchische Adressierungsstruktur des gleichen Wörterbuchartikels erweiterte hierarchische hybride Artikelmikrostruktur; deswegen wird statt des Terminus Angabenstruktur auch der synonyme Terminus Mikro- mit Adressierungsstruktur verwendet. Eine hierarchische hybride exhaustive Angabenstruktur eines Wörterbuchartikels ist die um die nichthierarchische Adressierungsstruktur des gleichen Wörterbuchartikels erweiterte hierarchische hybride Artikelkonstituentenstruktur. Die Kenntnis dessen, was unter Adressierung, unter Angabeadressierung, unter Angabezusatzadressierung und besonders unter einer artikelzugehörigen Adressierungsstruktur verstanden wird, muss hier vorausgesetzt werden (vgl. z.B. Wiegand 2006 und WLWF-1 2010).

Wir betrachten zunächst hierarchische hybride Angabenstrukturen. Verfügt man bereits über die konkrete und isomorphe abstrakte hierarchische hybride Artikelmikrostruktur sowie über die konkrete und isomorphe abstrakte Adressierungsstruktur eines Wörterbuchartikels, dann sind die artikel- 


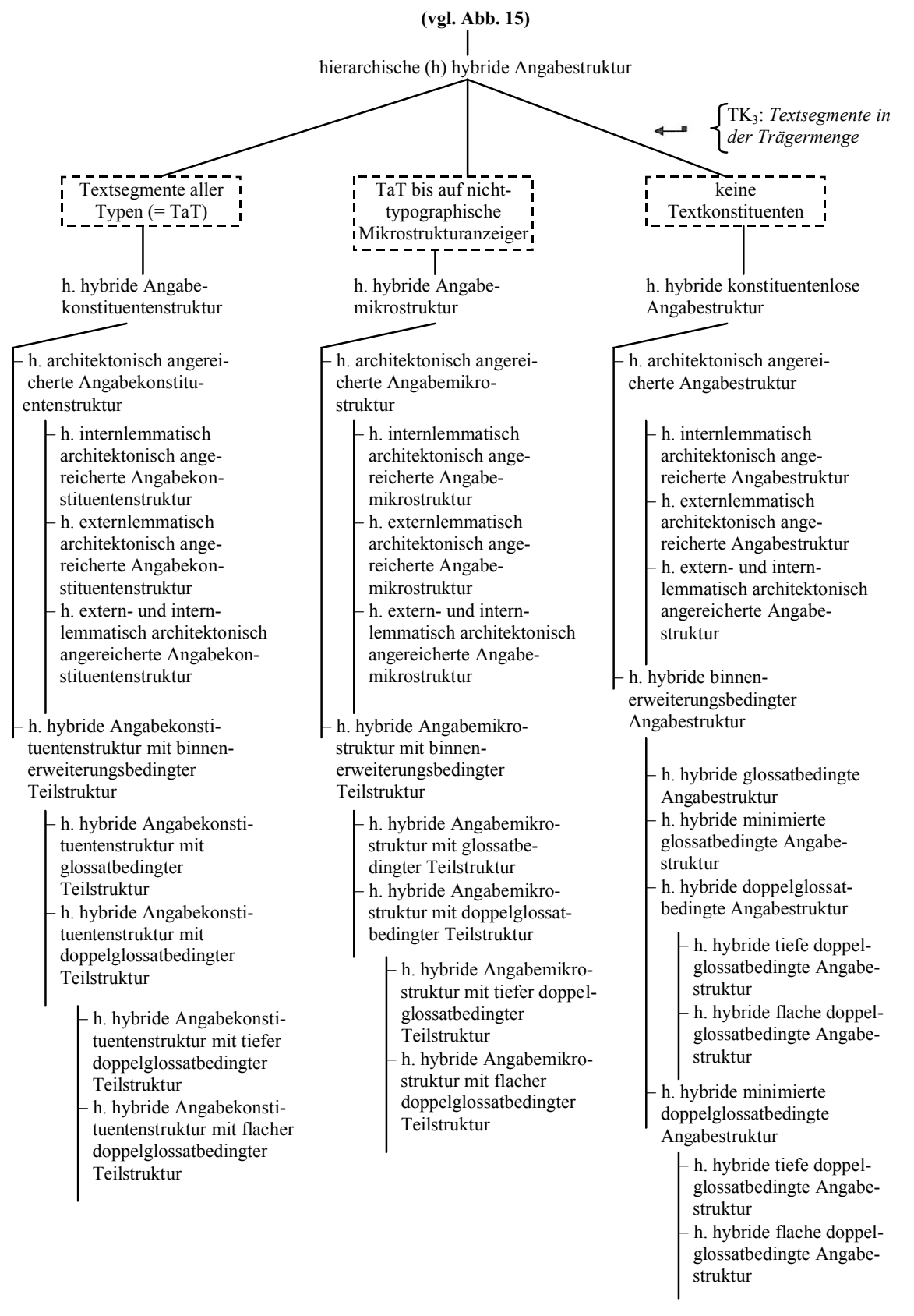

Abb. 20: Partiell erweitert kommentierter Typologiegraph zu einem Ausschnitt aus einer Typologie von hierarchischen hybriden Angabestrukturen Abkürzungen: $\mathrm{h}$ = hierarchisch; TK = Typologiekriterium; Darstellungskonventionen: „« - " bedeutet soviel wie die Anwendung des TK führt zu der Unterteilung 
zugehörigen konkrete und abstrakte Angabenstruktur dadurch erhältlich, dass man die beiden erstgenannten textuellen Strukturen zusammenfügt. Eine solche Strukturzusammenfügung lässt sich sowohl strikt formal als auch auf der Darstellungsebene vornehmen, wie es nachfolgende geschieht. Will man von der Darstellung einer hierarchischen hybriden Artikelmikrostruktur zur Darstellung einer hierarchischen hybriden Angabenstruktur übergehen, hat man mindestens die folgenden vier Darstellungsmethoden (vgl. Wiegand 2010):

(a) Man repräsentiert innerhalb des Strukturgraphen jede Adressierungsbeziehung (analog zu den Pfeildiagrammen bei der Darstellung von Adressierungsstrukturen) durch einen Pfeil, der vom adressierten Textsegment zu dessen Bezugsadresse führt.

(b) Man verwendet Adressensymbole, die die Bezugsadressen bezeichnen und die - getrennt durch einen fetten Mittenpunkt - hinter jedes Klassensymbol für eine Klasse von adressierten Textsegmenten gesetzt werden, das im Mikrostrukturgraphen als Knotenetikett fungiert. Ein entsprechendes erweitertes Knotenetikett hat z.B. die Form "MorA.S • LZGA" und ist - vereinfacht ausgedrückt - zu lesen wie an die LZGA adressierte MorA.S.

(c) Man verwendet Adressensymbole im Zusammenhang mit der Darstellungskonvention, dass bei adressenhomogenen Angaben die Verwendung der Adressensymbole bei allen Teilangaben der adressenhomogenen Mutterangabe unterbleibt, weil die Adressierungsbeziehungen erschließbar sind.

(d) Man repräsentiert innerhalb des Mikrostrukturgraphen Adressierungsbeziehungen durch Pfeile im Zusammenhang mit der Darstellungskonvention, dass bei adressenhomogenen Angaben nur bei der Mutterangabe ein Pfeil zur Bezugsadresse führt, aber keiner von den adressengleichen Teilangaben der Mutterangabe, weil diese erschließbar sind.

Für die Strukturdarstellung von hierarchischen reinen und hybriden Angabenstrukturen kann die die Darstellungskonvention in Geltung gesetzt werden, dass die Angabeadressierungsbeziehungen in der konkreten Struktur nicht explizit berücksichtigt werden, da sie aus der Darstellung in der abstrakten Struktur erschlossen werden können.

Die anschauliche Darstellungsmethode (a) eignet sich besonders zur Darstellung der hierarchischen reinen und hybriden Angabenstrukturen von Kurzartikeln sowie für die Darstellung solcher Kommentarangabenstrukturen (also Angabenteilstrukturen von artikelzugehörigen Angabenstrukturen), die zu einem Formkommentar gehören. In Abb. 21 findet sich die Darstellung der hierarchischen hybriden Kommentarangabenstruktur, die der Formkommentar von wa $\left(=\mathrm{fk}_{5}\right)$ in Abb. 1 aufweist nach der Darstellungsmethode (a) (vgl. Abb. 7). 


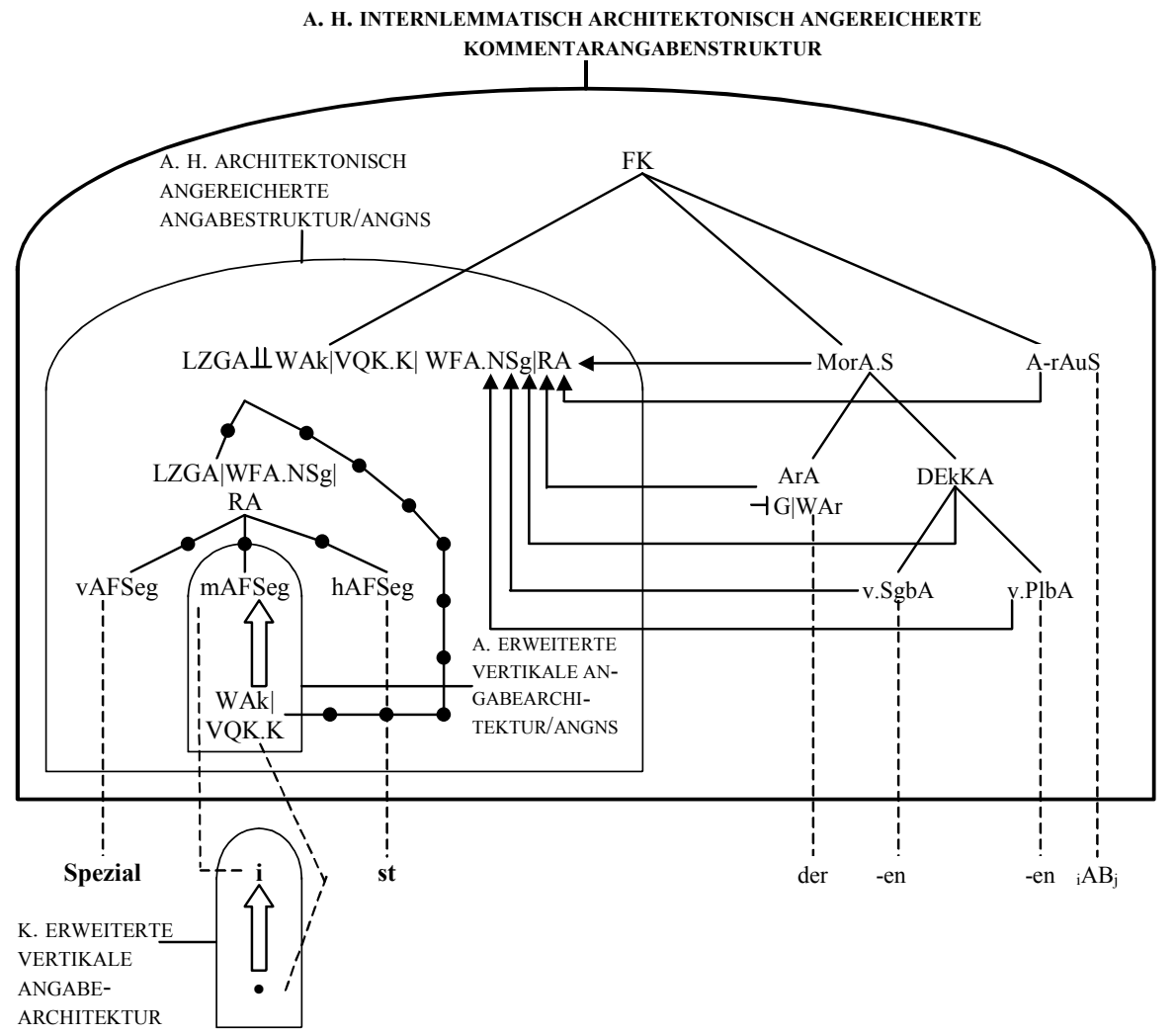

Abb. 21: Einfach kommentierter und mit architektonischen Komponenten angereicherter Strukturgraph zur konkreten (und isomorphen abstrakten) internlemmatisch architektonisch angereicherten Kommentarangabenstruktur, die $\mathrm{fk}_{5}$, der Formkommentar von wa $\mathrm{a}_{5}$ in Abb. 1, aufweist, ", $\mathrm{x} \Longrightarrow \mathrm{y}$ " bedeutet soviel wie $x$ ist unterhalb von $y$ und an $y$ hinaufadressiert, wenn der Pfeil nach oben zeigt.

Betrachtet man die in Abb. 21 dargestellte hierarchische Struktur unter dem Aspekt, dass sie eine Teilstruktur der artikelzugehörigen hierarchischen hybriden Angabenstruktur ist, wird sie auch hierarchische hybride linke Kernstruktur/AngnS genannt. Dass in Abb. 21 erweiterte vertikale Angabearchitekturen vorliegen, liegt daran, dass auf der Trägermenge der Angabearchitektur nicht nur eine unterhalb-Relation, sondern auch eine zweistellige asymmetrische und irreflexive Relation vom Typ der Hinaufadressierungsrelation definiert ist. Denn die Wortakzentkennzeichnung, die zugleich eine Vokalquantitätskennzeichnung zur Kürze und durch den Unterpunkt realisiert ist, ist an das Angabeformsegment $\mathbf{i}$ hinaufadressiert. 


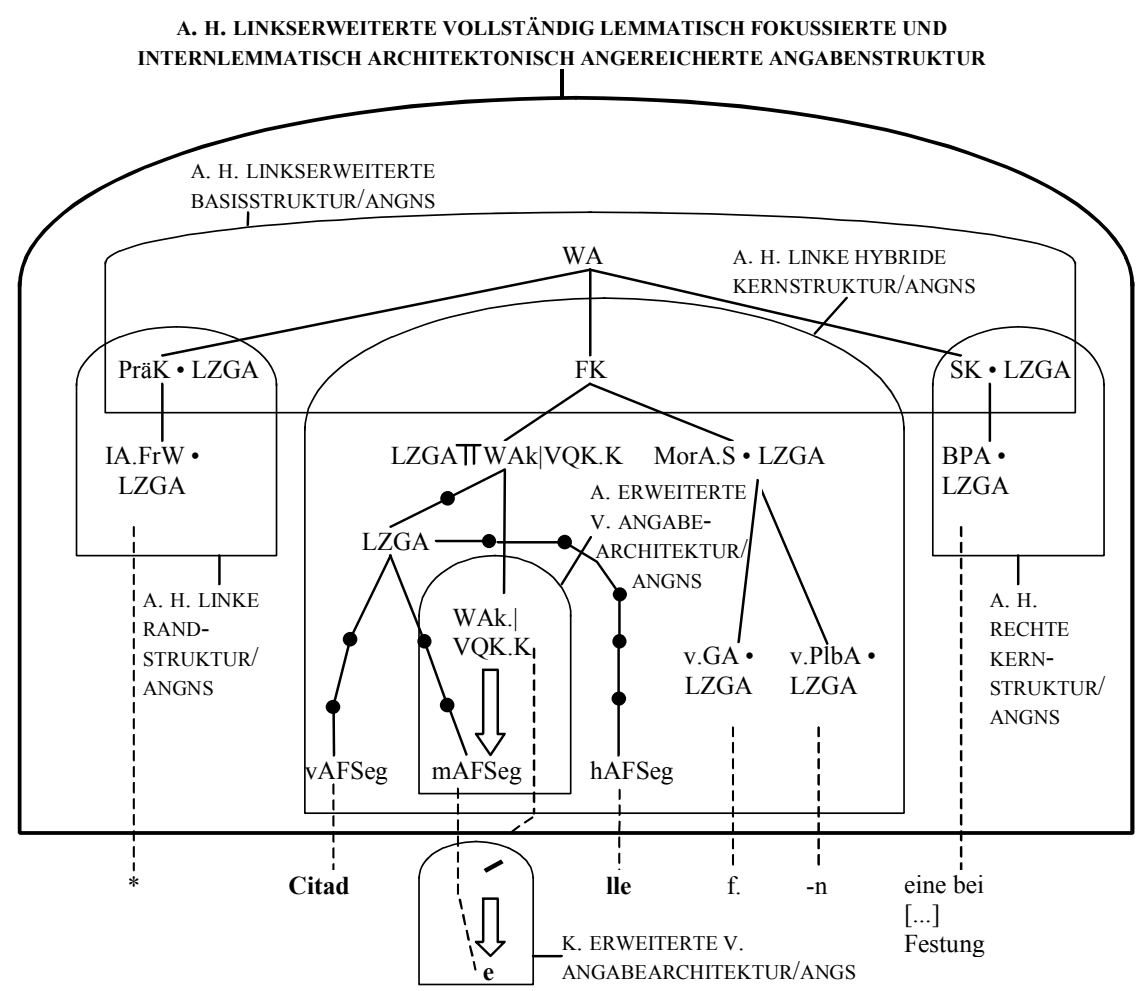

Abb. 22: Einfach kommentierter und mit architektonischen Komponenten angereicherter Strukturgraph zur abstrakten (und isomorphen konkreten) hierarchischen linkserweiterten vollständig lemmatisch fokussierten und internlemmatisch architektonisch angereicherten Angabenstruktur, die wa ${ }_{11}$ in Abb. 17 aufweist

Abkürzungen: PräK = Präkommentar; IA.FrW = Fremdwortidentifizierungsangabe (realisiert durch einen Asterisk); v.GA = verdichtete Genusangabe; Darstellungskonventionen: , $\mathrm{x} \Longrightarrow \mathrm{y}$ " bedeutet soviel wie $x$ ist oberhalb von $\mathrm{y}$ und an y hinabadressiert, wenn der Pfeil nach unten zeigt ", $\mathrm{u} \rightarrow \bullet-\mathrm{v}$ " bedeutet soviel wie $u$ ist ein Teil von $v$, mit ", $\mathrm{u}^{\prime \prime}$ als Variable für Textsegmente, die keine Textkonstituenten sind.

Verfügt man nicht über die hierarchische hybride Artikelmikrostruktur eines Artikels sowie zugleich über die artikelzugehörige nichthierarchische Adressierungsstruktur und möchte die hierarchische hybride Angabenstruktur eines Wörterbuchartikels wa $a_{x}$ bilden, dann muss man nach der Bildung der elementenheterogenen Trägermenge, die die gleichen Elemente aufweist als wolle man die hierarchische hybride Artikelmikrostruktur $\mathrm{zu} \mathrm{wa}_{x}$ bilden, auf dieser Trägermenge zwei Relationen der uns bekannten Typen definieren, nämlich eine Relation vom Typ der partitiven und eine vom Typ der Präzedenzrelation, 
sowie weiterhin auf den geeigneten Teilmengen der Trägermenge eine zweistellige, asymmetrische und irreflexive Relation vom Typ der Angabeadressierungsrelation mit dem Relationsterm $x$ ist adressiert an $y$ (mit " $x^{\text {" als Variable }}$ für adressierte Angaben und " $y$ " als Variable für bezugsadressentragende Angaben und damit für Bezugsadressen) sowie schließlich eine zweistellige, asymmetrische und irreflexive Relation vom Typ der Angabezusatzadressierungsrelation mit dem Relationsterm $u$ ist adressiert an $v$ (mit " $u$ " als Variable für funktionale Angabezusätze und " $v^{\prime \prime}$ als Variable für die Träger von Angabezusatzadressen und damit für Angabezusatzadressen). Das Ergebnis ist dann eine hierarchische hybride Angabenstruktur wie die in Abb. 22 dargestellte von wa 11 .

Im Folgenden betrachten wir ein Beispiel für hierarchische hybride exhaustive Angabenstrukturen eines Wörterbuchartikels anhand von wa in Abb. 1 und auf der Basis von Abb. 14; das bedeutet, dass (aus Platzgründen) der Übergang von der Artikelkonstituenten - zur artikelzugehörigen Angabenkonstituentenstruktur auch hier lediglich auf der Darstellungsebene vorgenommen wird. Angewandt wird die oben erläuterte Darstellungsmethode (b), so dass die artikelinternen Angabeadressierungsbeziehungen dadurch repräsentiert wird, dass hinter jedes Klassensymbol für eine Klasse von adressierten Textsegmenten hinter einem fetten Mittenpunkt das Adressensymbol steht. Während in $\mathrm{wa}_{11}$ in Abb. 17 die Adressierungskonstellation der vollständig lemmatischen Adressierung mit internlemmatischer Adressierung (vgl. Wiegand 2006: 230ff $u$. WLWF-1 2010) vorliegt (jede internlemmatische Adressierung ist eine Angabezusatzadressierung), so dass alle adressierungsfähigen Angaben an die als lemmatische Substantivangabe ausgeprägte Lemmazeichengestaltangabe adressiert sind, und deswegen nur das Adressensymbol LZGA vorkommt (vgl. Abb. 22), liegt in $\mathrm{wa}_{6}$ in Abb. 1 die Adressierungskonstellation der partiell lemmatischen Adressierung mit externlemmatischer Angabezusatzadressierung vor. Das bedeutet auch, dass mehrere verschiedene Adressensymbole auftreten, denn im Postkommentar zu Phraseologie sind nicht alle Angaben an die Lemmazeichengestaltangabe adressiert, und auch die Glossate sind nichtlemmatisch adressiert. Es ist klar, dass die Klassensymbole für die nichttypographischen Mikrostrukturanzeiger (z.B. TS) nicht mit einem Adressensymbol versehen werden, da nichttypographische Mikrostrukturanzeiger nicht adressiert sind. Ein Ausschnitt aus der abstrakten (und isomorphen konkreten) partiell lemmatisch fokussierten hybriden exhaustiven Angabenstruktur mit tiefer doppelglossatbedingter Teilstruktur findet sich in Abb. 23.

Im Folgenden betrachten wir die in Abb. 23a nur markierte und nicht analysierte hybride tiefe glossatbedingte Angabe- mit Adressierungsstruktur, die die verdichtete und um ein semantisches Doppelglossat binnenerweiterte Kompetenzbeispielangabe "das hat viel S (Mühe, Anstrengung) gekostet" (= $\mathrm{a}_{6}$ ) in wa $\mathrm{a}_{6}$ in Abb. 1 aufweist und die in Abb. 23b dargestellt ist. 


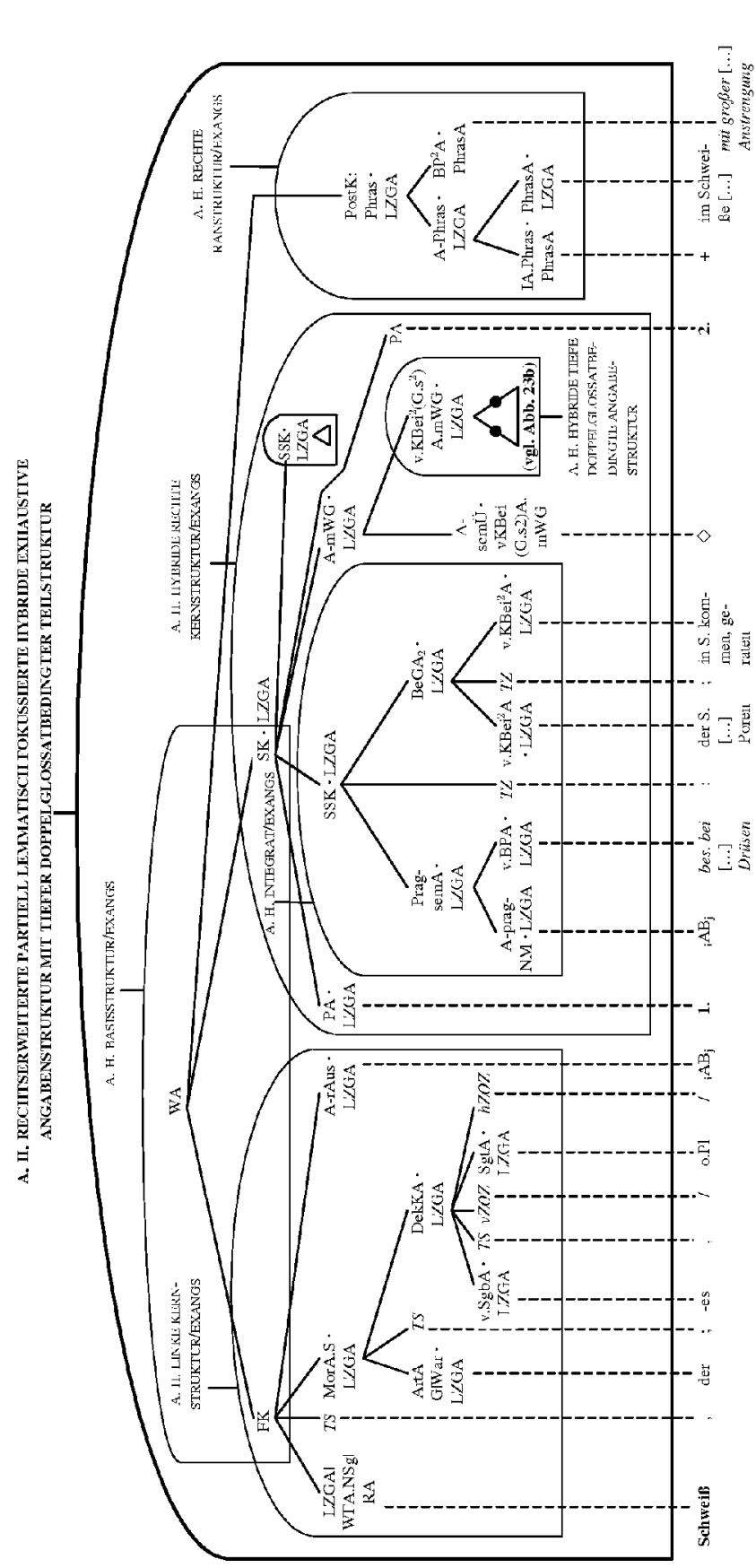

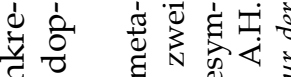

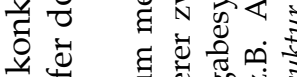

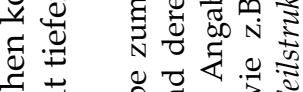

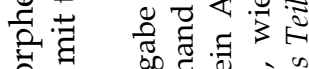

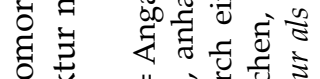
.0.

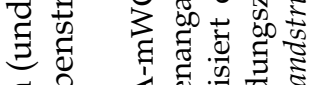
ฮ

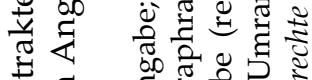

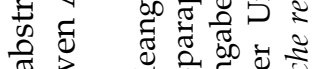
त.

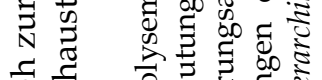
중

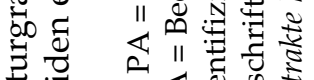

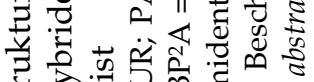
की

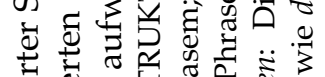

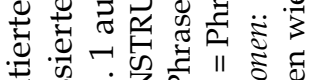

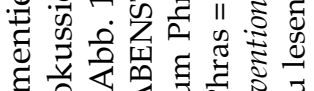

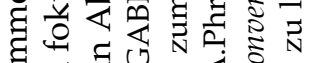

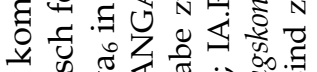

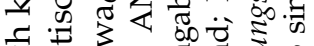

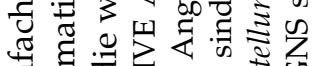

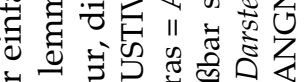

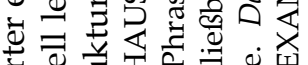

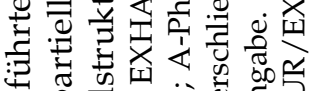
क्ष.

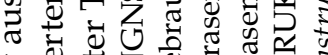
我.

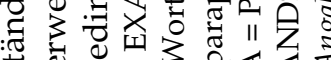
क्ञ

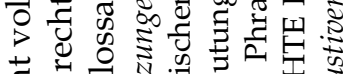

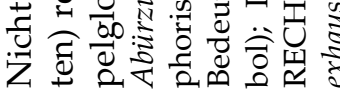
$\ddot{0}$
$\stackrel{0}{0}$
$\dot{0}$
$\dot{0}$ 


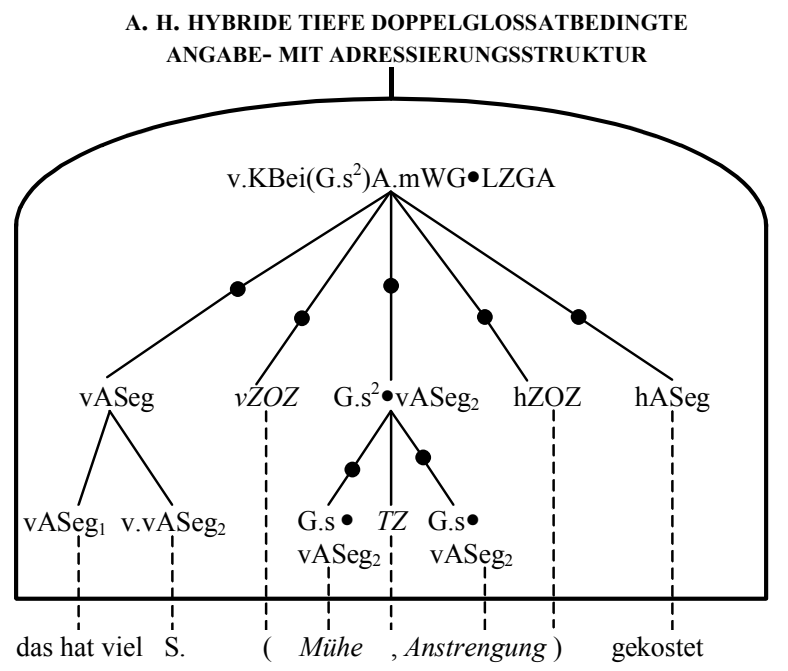

Abb. 23b: Einfach kommentierter Strukturgraph zur abstrakten (und isomorphen konkreten) tiefen doppelglossatbedingten Angabe- mit Adressierungsstruktur

Abkürzungen: v.vASeg = verdichtetes vorderes Angabesegment

Oben wurde festgestellt, dass Angabenstrukturen textuelle Strukturen von ganzen Wörterbuchartikeln sind. Es gibt aber auch Angaben, wie z.B. $a_{6}$, innerhalb derer Adressierungsbeziehungen auftreten; so ist in $\mathrm{a}_{6}$ das erste semantische Binnenglossat "Mühe" adjazent links an "S." adressiert, und das zweite semantische Binnenglossat ist nichtadjazent links an "S." linksadressiert. Um nicht einen zu leicht missverständlichen Terminus wie angabeinterne Angabenstruktur verwenden zu müssen, wird daher von Angabemikrostruktur mit Adressierungsstruktur oder verkürzt von Angabe- mit Adressierungsstruktur gesprochen (in Analogie zu dem oben erwähnten terminologischen Synonym Mikromit Adressierungsstruktur zu Angabenstruktur.

Um die in Abb. 23b dargestellte Angabe- mit Adressierungsstruktur, die $\mathrm{zu}_{6}$ gehört, bilden zu können, muss das vordere Angabesegment ", das hat viel S." weiter nichtfunktional-positional segmentiert werden, damit als Teilsegment des verdichteten vorderen Angabeformsegments das "S." isoliert wird, das Adressenträger der Bezugsadressen S| der beiden semantischen Binnenglossate ist. Erst dann kann auf den zuständigen Trägermengen für die konkrete und abstrakte Strukturausprägung neben der partitiven und der Präzedenzrelation eine zweistellige asymmetrische und irreflexiven Relation vom Typ der Angabezusatzadressierungsrelation definiert werden, so dass die in Abb. 23b dargestellte hybride tiefe doppelglossatbedingte Angabe- mit Adressierungsstruktur erhältlich ist. 
Abschließend sei zu den artikelinternen hierarchischen reinen und hybriden Angabenstrukturen und exhaustiven Angabenstrukturen bemerkt, dass sie für theoriegeleitetes Verfassen von Benutzungshinweisen die am besten geeigneten artikelzugehörigen textuellen Strukturen sind, weil sie - anders als die hierarchischen reinen und hybriden Artikelkonstituenten- und Artikelmikrostrukturen - Auskunft darüber geben, auf welche Angabegegenstände anderer artikelinterner Angaben die adressierten Angaben und adressierten funktionalen Angabezusätze zu beziehen sind, wenn ein Benutzer anhand artikelinterner Angaben und funktionaler Angabezusätze eine lexikographische Information (verstanden als punktuelles Wissen und damit als kognitives Phänomen) erschließen möchte (vgl. dazu Wiegand 2000b).

\section{Hybride textuelle Einheiten: eine kleine Auswahl}

Wir wissen bereits, dass die funktional-positionale Segmentation von Textsegmenten, die selbst das Ergebnis einer nichtfunktionalen Segmentation und damit keine Textkonstituenten sind, wie das (nichtelementare) semantische Doppelglossat "Mühe, Anstrengung", hybride Textkonstituenten sind, da sie von zweierlei Herkunft sind (vgl. die Bedeutungsparaphrasenangabe des Duden${ }^{4} \mathrm{GFWb}$ in 1.).

Potenziell nichtelementare Glossate jeder Art und nicht nur Doppelglossate haben, wenn sie im Kotext eines Wörterbuchartikels oder in dem eines der anderen akzessiven Einträge auftreten, die Eigenschaft aller Binnenglossate: Sie sind funktional-positional nicht isolierbar, weil bei der Segmentation der Angabe, die sie binnenerweitern, nichtfunktionale Textsegmente, nämlich vordere und hintere Angabesegmente auftreten. Damit sind sie funktionale Angabezusätze ohne Textkonstituentenstatus und weisen die kriteriale Eigenschaft der Elemente dieser Textsegmentklasse auf. Sind sie aber nichtfunktional-positional isoliert, dann weisen sie kriteriale Eigenschaft der Textkonstituenten auf: Sie sind dann aktuell nichtelementar und damit restfrei in funktionale Textsegmente durch eine Anwendung der Methode der funktional-positionalen Segmentation zerlegbar.

Dies sei im Folgenden anhand zweier anderer Typen von potenziell nichtelementaren Binnenglossaten gezeigt (vgl. Wiegand 2010a). Im Artikel wa 10 in Abb. 17 findet sich folgende elementare Angabe: „eine e (ANT große) Pause“ $\left(=\mathrm{a}_{10}\right)$. Es handelt sich um eine verdichtete Kompetenzbeispielangabe, die um ein semantisches Binnenglossat erweitert ist, bei dem es sich um eine Kennzeichnung zur Antonymie handelt, die aus "ANT", einer verdichteten Antonymidentifizierungskennzeichnung, sowie aus "große", einem Antonymhinweis besteht. Um die konkrete hierarchische glossatbedingte Angabestruktur von $\mathrm{a}_{10}$ bilden zu können, muss $\mathrm{a}_{10}$ nichtfunktional-positional segmentiert werden, und zwar wie folgt: eine e | ( | ANT große | ) | Pause (mit "|" als Segmentationsfugenmarkierung). Es gelten folgende Aussagen: eine $\sim$ e $\in$ vASeg; $(\epsilon$ vZOZ; ANT große $\in$ mASeg; ) $\in h Z O Z$ und Pause $\in$ gASeg. Alle fünf Textseg- 
mente sind keine Textkonstituenten. Das mittlere Angabesegment (mASeg), die isolierte Kennzeichnung zu Antonymie, lässt sich restfrei funktional-positional in zwei Angaben segmentieren und ist damit - wie das Doppelglossat aus wa 6 - als hybride Textkonstituente einzustufen. Die abstrakte (und isomorphe konkrete) hierarchische tiefe glossatbedingte Angabestruktur findet sich in Abb. 24.

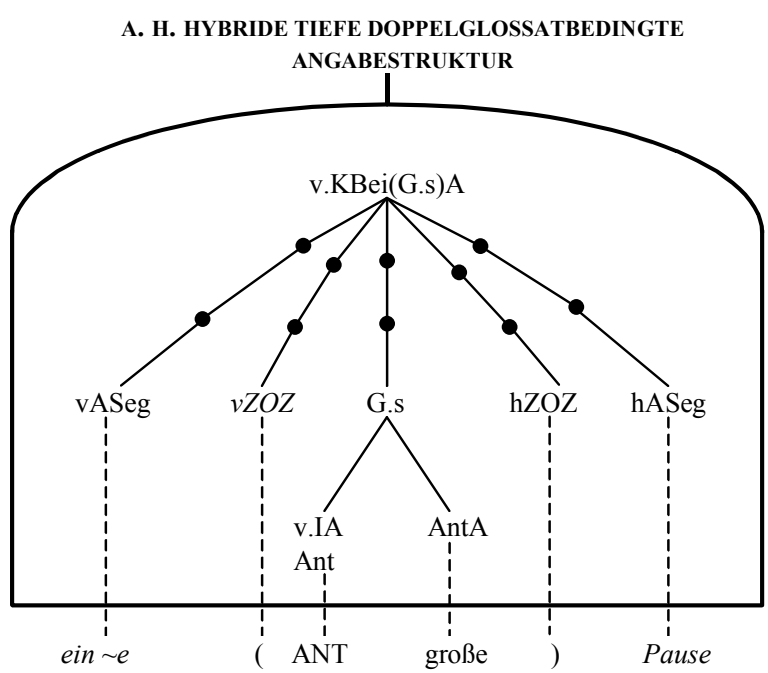

Abb. 24: Einfach kommentierter Strukturgraph zur abstrakten (und isomorphen konkreten) hierarchischen hybriden glossatbedingten Angabestruktur, konzipiert als tiefe Struktur Abkürzungen: v.IA.Ant = verdichtete Antonymenidentifizierungsangabe; Ant $\mathrm{A}=$ Antonymangabe

Auch die um eine Kennzeichnung zur Synonymie binnenerweiterte Kompetenzbeispielangabe "ein es (",SYN 'niedriges' 2") Gehalt" aus wa $\mathrm{a}_{8}$ in Abb. 17, deren semantisches Binnenglossat aus einer verdichteten Synonymidentifizierungsangabe "SYN" und aus einem um eine Bedeutungsidentifizierungskennzeichnung rechtserweiterten Synonymhinweis „'niedriges' 2“ besteht, lässt sich analog zum vorhergehenden Beispiel analysieren, so dass "Syn 'niedriges' 2" als hybride Textkonstituente einzustufen ist.

Als letztes Beispiel sei anhand von wa $a_{9}$ und wa ${ }_{10}$ in Abb. 25 erläutert, was unter einer hybriden Verweiskennzeichnung zu verstehen ist.

wa9: Dorf, das; -s, Dörfer.

+ die $>$ Kirche im D. lassen

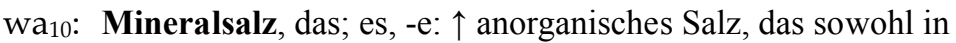
der Natur vorkommt als auch künstlich hergestellt wird.

Abb. 25: Wörterbuchartikel wa9 (gekürzt) aus HWDG und wa 10 (gekürzt) aus Duden-4GFWb 
Funktionale Angabezusätze vom Typ der Verweiskennzeichnung, wie z.B. "> Kirche", in der verdichteten verweisvermittelnden Phrasemangabe in was in Abb. 25, die nichthybrid sind, weisen eine Verweisbeziehungskennzeichnung auf - in wa9 realisiert durch den Schrägpfeil - die im Unterschied zu erweiternden Verweisbeziehungsangaben, wie " $\uparrow$ " in wa 10 $_{10}$, funktional-positional nicht isolierbar sind. In wa 10 ist die Definiensangabe um den Hochpfeil "^^" linkserweitert. Durch diese Linkserweiterung ergibt sich die usuelle Verweiskennzeichnung,$\uparrow$ anorganisches Salz"; diese ist als ganzes Textsegment nicht funktional-positional isolierbar. Bei einer funktional-positionalen Segmentation von $w_{10}$ ist der Hochpfeil jedoch eine eigenständige Textkonstituente, mit der eine erweiternde Verweisbeziehungsangabe gegeben ist, und zwar ein linkserweiternde. Dies bedeutet: Verweiskennzeichnungen mit linkserweiternden Verweisbeziehungsangaben sind hybride funktionale Angabenzusätze, die keine Textkonstituenten sind.

\section{Schlussbemerkung}

Wörterbuchartikeln, die mindestens einen funktionalen Angabezusatz aufweisen, können - wie gezeigt wurde - reine und hybride textuelle Strukturen vom Typ der Artikelkonstituenten- und vom Typ der Artikelmikrostruktur, weiterhin solche vom Typ der artikelinternen Angabenstruktur und schließlich solche vom Typ der artikelinternen exhaustiven Angabenstruktur zugewiesen werden. Entsprechendes gilt für erweiterte Angaben und für nichtelementare um funktionale Angabezusätze erweiterte Angaben. In allen Fällen sind die hybriden textuellen Strukturen in einem höheren Grad explizit, und vor allen Dingen erlauben sie leichter eine genaue quantitative Analyse der propositionalen Gehalte, die der Lexikograph dem Benutzer vermitteln möchte. Dies gilt besonders für die hybriden Artikelmikrostrukturen, die als Distributionsstrukturen für alle funktionalen Textsegmente mit Angabefunktion im Wörterbuchartikel verstanden werden müssen. Um ein Wörterbuch mit hybriden Strukturen und Einheiten erfolgreich benutzen zu können, muss das Wissen des kundigen Benutzers (i.S.v. Wiegand 1998: 506) größer sein als wenn nur reine Strukturen gegeben sind.

\section{Literatur}

Bergenholtz, Henning, Sven Tarp und Herbert Ernst Wiegand. 1999. Datendistributionsstrukturen, Makro- und Mikrostrukturen in neueren Fachwörterbüchern. Hoffmann, L., H. Kalverkämper und H.E. Wiegand (Hrsg.). 1999. Fachsprachen. Ein internationales Handbuch zur Fachsprachenforschung und Terminologiewissenschaft/Languages for Special Purposes. An International Handbook of Special-Language and Terminology Research: 1762-1832. Handbücher zur Sprachund Kommunikationswissenschaft 14.2. Berlin/New York: Walter de Gruyter.

Bourbaki, Nicolas. 1957. Éléments de mathématique. I. Les structures fondamentales de l'analyse, livre I: Théorie des ensembles, chapitre 4: Structures. Paris: Hermann [engl. Fassung 1968]. 
DGWDaF 2000 = Kempcke, G. (Hrsg.). Wörterbuch Deutsch als Fremdsprache. Berlin/New York: Walter de Gruyter.

Duden- ${ }^{4}$ GFWb 2007 = Das grosse Fremdwörterbuch. Herkunft und Bedeutung der Fremdwörter. 4., aktual. Aufl. Hrsg. u. bearb. vom Wiss. Rat der Dudenredaktion. Mannheim [usw.]: Dudenverlag.

Hausmann, Franz Josef, Oskar Reichmann, Herbert Ernst Wiegand und Zgusta Ladislav (Hrsg.). 1989-1991. Wörterbücher. Ein internationales Handbuch zur Lexikographie/Dictionaries. An International Encyclopedia of Lexicography/Dictionnaires. Encyclopédie internationale de lexicographie. 3 Teilbände. Berlin/New York: Walter de Gruyter.

Hollós, Zita. 2001. Német-Magyar suliszótár. Szeged: Grimm Könyvkiadó.

HWDG 1984 = Handwörterbuch der deutschen Gegenwartssprache. In zwei Bänden. Von einem Autorenkollektiv unter der Leitung v. G. Kempcke [...] Bd. 1: A-K; Bd. 2: L-Z. Berlin: AkademieVerlag.

Sanders-WDS 1969 = Daniel Sanders: Wörterbuch der deutschen Sprache. Mit Belegen von Luther bis auf die Gegenwart. 1. Bd. A-K. 2., unv. Abdruck. Leipzig: O. Wigand, 1876 [Nachdruck Hildesheim 1969. Mit einer Einführung und Bibliographie v. Werner Betz].

Weigand, Friedrich Ludwig Karl. 1873. Deutsches Wörterbuch. 1. Bd. A-L. 2., verb.u. verm. Auflage. Gießen: J. Richer'sche Buchhandlung.

Wiegand, Herbert Ernst. 1989. Der Begriff der Mikrostruktur. Geschichte, Probleme, Perspektiven. Hausmann, F.J., O. Reichmann, H.E. Wiegand and L. Zgusta (Hrsg.) 1989-1991: 409-462.

Wiegand, Herbert Ernst. 1989a. Arten von Mikrostrukturen im allgemeinen einsprachigen Wörterbuch. Hausmann, F.J., O. Reichmann, H.E. Wiegand and L. Zgusta (Hrsg.) 1989-1991: 462501.

Wiegand, Herbert Ernst. 1990. Printed Dictionaries and their Parts as Texts. An Overview on Recent Research as an Introduction. Lexicographica 6: 1-126 [Auch in Wiegand 2000: 951-1062].

Wiegand, Herbert Ernst. 1998. Wörterbuchforschung. Untersuchungen zur Wörterbuchbenutzung, zur Theorie, Geschichte, Kritik und Automatisierung der Lexikographie. 1. Teilbd. Mit 159 Abbildungen im Text. Berlin/New York: Walter de Gruyter.

Wiegand, Herbert Ernst. 2000. Kammerer, Matthias und Werner Wolski (Hrsg.). Kleine Schriften. Eine Auswahl aus den Jahren 1970 bis 1999 in zwei Bänden. Bd. 1: 1970-1988; Bd. 2: 1988-1999. Berlin/New York: Walter de Gruyter.

Wiegand, Herbert Ernst. 2000a. Wissen, Wissensrepräsentation und Printwörterbücher. Heid, U., St. Evert, E. Lehmann und Ch. Rohrer (Hrsg.). 2000. Proceedings of the Ninth EURALEX Intenational Congress. EURALEX 2000. Stuttgart, Germany, August 8-12.2000: 15-38. Stuttgart: Institut für Maschinelle Sprachverarbeitung, Universität Stuttgart.

Wiegand, Herbert Ernst. 2001. Sprachkontaktwörterbücher, Typen, Funktionen, Strukturen. Igla B., P. Petkov und H.E. Wiegand (Hrsg.). 2001. Theoretische und praktische Probleme der Lexikographie. 1. Internationales Kolloquium zur Wörterbuchforschung am Institut Germanicum der St. Kliment Ohridski-Universität, Sofia, 7. bis 8. Juli 2000: 115-224. Germanistische Linguistik 161-162: Hildesheim/Zürich/New York: Georg Olms Verlag.

Wiegand, Herbert Ernst. 2002. Über textuelle Strukturen der Wörterbuchartikel und Artikelnischen im de Gruyter Wörterbuch Deutsch als Fremdsprache. Zugleich ein Beitrag zur Weiterentwicklung einer Theorie der Wörterbuchform. Wiegand, H.E. (Hrsg.). 2002. Perspektiven der pädagogischen Lexikographie des Deutschen II. Untersuchungen anhand des de Gruyter Wörterbuchs Deutsch als Fremdsprache: 497-595. Lexicographica. Series Maior 110. Tübingen: Max Niemeyer Verlag. 
Wiegand, Herbert Ernst. 2003. Überlegungen zur Typologie von Wörterbuchartikeln in Printwörterbüchern. Ein Beitrag zur Theorie der Wörterbuchform. Lexicographica 19: 169-313.

Wiegand, Herbert Ernst. 2005. Angaben, funktionale Angabezusätze, Angabetexte, Angabestrukturen, Strukturanzeiger, Kommentare und mehr. Ein Beitrag zur Theorie der Wörterbuchform. Lexicographica 21: 202-379.

Wiegand, Herbert Ernst. 2006. Adressierung in Printwörterbüchern. Präzisierungen und weiterführende Überlegungen. Lexikographica 22: 187-261.

Wiegand, Herbert Ernst. 2009. Nichtnatürlich über natürliche Sprache schreiben. Zu einigen formalen Aspekten von Wörterbuchartikeln. Heidelberger Jahrbücher 53: 247-285.

Wiegand, Herbert Ernst. 2009a. Hybrid Text Constituent Structures of Dictionary Articles. A Contribution of the Expansion of the Theory of Textual Dictionary Structures. Nielsen, S. und S. Tarp (Hrsg.). 2009. Lexicography in the 21st Century. In Honour of Henning Bergenholtz: 63-89. Terminology and Lexicography Research and Practice 12. Amsterdam/Philadelphia: John Benjamins.

Wiegand, Herbert Ernst. 2010. Zur Methodologie der Wörterbuchforschung: Ausgewählte wörterbuchbezogene Analyse- und Darstellungsmethoden. Lexicographica 26. [Erscheint].

Wiegand, Herbert Ernst. 2010a. Semantik, Pragmatik und Wörterbuchform in einsprachigen Wörterbüchern. Zeitschrift für germanistische Linguistik 38(3). [Erscheint].

Wiegand, Herbert Ernst und $\mathbf{M}^{\mathrm{a}}$ Teresa Fuentes Morán. 2009. Estructuras lexicográficas. Aspectos centrales de una teoría de la forma del diccionario. Granada: Tragacanto.

WLWF-1. 2010 = Wörterbuch zur Lexikographie und Wörterbuchforschung. Dictionary of Lexicography and Dictionary Research. Mit englischen Übersetzungen der Umtexte und Definitionen sowie Äquivalenten in neun Sprachen/With English Translations of the Outer Texts and Definitions as well as Equivalents in Nine Languages. Hrsg. u. Bearb. v./Ed. and compiled by Herbert Ernst Wiegand, Michael Beißwenger, Rufus H. Gouws, Matthias Kammerer, Angelika Storrer, Werner Wolski unter Mitarbeit von/with the collaboration of Ekaterina Butina-Koller (Russisch/Russian), Rute Costa (Portugiesisch/Portuguese), Ma Teresa Fuentes Morán (Spanisch/Spanish), Laura Giacomini (Italienisch/Italian), Rufus H. Gouws (Afrikaans; Englisch/English), Franz Josef Hausmann und Maria Hegner (Französisch/French), Regina Hessky und Zita Hollós (Ungarisch/Hungarian), Pavel Petkov (Bulgarisch/Bulgarian), Giovanni Rovere (Italienisch/ Italian), Stefan J. Schierholz (Portugiesisch/Portuguese), Maria Smit (Englisch/English). 4 Bde./4 Vols. 1. Bd.: Systematische Einführung/Vol. I: Systematic Introduction. A-C. Mit 120 Grafiken von/with 120 Figures by Matthias Kammerer. Berlin/New York: Walter de Gruyter. [Mit/with CD-ROM]. 